\title{
Formen und Funktionen von Interrogationen. Fragen in grammatischen Beschreibungen, empirischen Befunden und Lehrwerken für Deutsch als Fremdsprache*
}

\author{
Martina Rost-Roth (Augsburg)
}

\begin{abstract}
Analysis of how questions are treated in grammar books shows that the main focus is on word order phenomena. At the same time, statements are often made about functions or the relationship between form and function; these are not always consistent with more systematic approaches or with empirical findings. Empirical analyses of spoken language reveal a very complex picture; comparison of the question realizations of native speakers and non-native speakers shows that the questions produced by non-native speakers are limited in various respects. Moreover, analysis of textbooks shows that some relationships between form and function are underrepresented.
\end{abstract}

\section{$1 \quad$ Einleitung}

Interrogationen sind sowohl in Hinblick auf Formen als auch Funktionen äußerst vielfältig. Schon in der Sprechhandlungstheorie haben sie aufgrund ihres Form-Funktionsverhältnisses besonderes Interesse gefunden und in syntaktischen Theoriebildungen bis hin zur Zweitspracherwerbsforschung sind Fragen im Deutschen aufgrund diverser Wortstellungsregularitäten von besonderem Interesse (Verb-Erst bzw. Inversion, Position bzw. Bewegung des Fragewortes in Satzanfangsstellung). Pragmatisch interessieren Fragen beispielsweise als Möglichkeiten zur Umsetzung von Höflichkeitsprinzipien über Indirektheit bei Bitten und Aufforderungen, zudem können sie insbesondere als Nachfragen in sehr unterschiedlichen Funktionen eingesetzt werden - womit nur einige Beispiele genannt seien. In der Zweit- und Fremdsprachendidaktik stellen Fragen einen zentralen pragmatischen Bereich dar, der seit der sog. kommunikativen Wende verstärkt Aufmerksamkeit erfährt. Dennoch zeigt sich bei der Betrachtung von Lehrwerken, dass bestimmte Form-Funktions-Verhältnisse unterrepräsentiert sind, und komplexe Fragerealisierungen, die insbesondere zur Umsetzung von höflichen Bitten und Aufforderungen und Realisierung von Höflichkeitsprinzipien eingesetzt werden können, noch unterrepräsentiert sind.

Da entsprechende Beobachtungen bereits auf einer Reihe eigener und anderer Untersuchungen aufbauen, werden die für den Beitrag zentralen Aussagen zunächst als Thesen formuliert und dann unter Verweis auf die Analysen dieser Untersuchungen begründet.

\section{Analysen und Befunde}

Die übergreifende und vergleichende Befassung mit Interrogationen in Grammatiken, sprachwissenschaftlichen Beschreibungen und Lehrwerken im Bereich Deutsch als Zweit-

\footnotetext{
* Den Herausgebern gilt mein Dank für gründliches Korrekturlesen.
} 
sprache sowie eigene korpusbezogene empirische Befunde haben zu Ergebnissen geführt, die sich in den folgenden Thesen zusammenfassen lassen:

(1) Fragen zeigen ein komplexes Form-Funktions-Verhältnis (Satzmodus - Sprechhandlung).

(2) Beschreibungen von Fragen in Grammatiken fallen in Bezug auf Form-FunktionsBezüge oft unstimmig aus.

(3) Empirische Analysen zu gesprochener Sprache zeigen, dass vorliegende Beschreibungen Form-Funktions-Bezüge oft nicht ausreichend erfassen und das Spektrum an Fragerealisierungen nicht voll abbilden.

(4) Formen und Funktionen von Fragen lassen sich systematischer beschreiben.

(5) Für Zweit- und Fremdspracherwerb stellen sich besondere Probleme in Hinblick auf (komplexere) Fragen.

(6) Die Systematisierung von Frage-Formen und -Funktionen ist auch in Hinblick auf eine kommunikativ-pragmatisch orientierte Didaktik von Bedeutung.

(7) Bestimmte Bezüge von Frage-Formen und -Funktionen sind (auch) in (neueren) Lehrwerken unterrepräsentiert.

\section{ad (1) Fragen zeigen ein komplexes Form-Funktions-Verhältnis (Satzmodus - Sprech- handlung)}

Fragen im Sinne von Satzmodus bzw. Satzart können nicht nur in Fragefunktion, sondern auch in anderen Funktionen wie z. B. Aufforderungen oder Vorwürfen eingesetzt werden. Umgekehrt können Fragefunktionen auch durch andere Satzarten und Modi wie z. B. Aussagemodus (wie z. B. über Formulierungen wie ich möchte fragen..., ich weiß nicht, ob...) realisiert werden.

Aufgrund der Komplexität des Form-Funktions-Verhältnisses sind auch die Bereiche, mit denen sich die Grammatikschreibung und Sprachwissenschaft befasst hat, entsprechend weit gefächert. ${ }^{1}$ In grammatischen Beschreibungen und Referenzgrammatiken steht die Beschreibung von syntaktischen Aspekten im Vordergrund: Hier interessieren vor allem die Syntax über Verbpositionen und Positionen des Fragewortes sowie Aspekte der Modusmarkierung (vgl. Burkhardt 1986, Altmann 1987, Brandt 1992, Hentschel 1998). In der Sprachwissenschaft findet zudem eine intensive Auseinandersetzung mit den formalen Besonderheiten bzw. Merkmalen von Echofragen wie Verb-Zweit und Fragewort-Positionen in situ statt (vgl. Klein 1982, Meibauer 1987, Reis/Rosengren 1991, Roberts 2007). Eine Auseinandersetzung mit Funktionen von Fragen hat vor allem in der Sprechakttheorie und Pragmatik stattgefunden. Bereits Austin (1962) und Searle (1974) befassen sich intensiv mit Fragefunktionen (type of illocutionary act, felicity conditions, indirect speech acts). In neueren Entwicklungen der Relevanztheorie spielen wiederum Echofragen und ironische Fragen eine besondere Rolle, die in anderen Ansätzen Beschreibungsprobleme aufwerfen (vgl. Wilson/Sperber 1988; Sperber/Wilson 1995). In der Konversationsanalyse und in gesprächsanalytischen Ansätzen hat eine intensive Auseinandersetzung mit Fragen und sequenziellen Aspekten stattgefunden. Auch aus der Zweitspracherwerbsforschung liegen aufschlussreiche Befunde zu Formen und Funktionen von Fragen vor (zu früheren Untersuchungen vgl. Wode 1976, Felix 1982, Clah-

\footnotetext{
1 Zum Form-Funktions-Verhältnis von Interrogationen vgl. z. B. Burkhardt (1986) oder Rehbein (1999: 105), der zwischen "der Handlung der Frage und dem Interrogativ als einem Mittel zu deren Realisierung" unterscheidet, wobei sprachliche Markierungen als Modusrealisierungen behandelt werden.
} 
Martina Rost-Roth: Formen und Funktionen von Interrogationen

sen/Meisel/Pienemann 1983, speziell zum Form-Funktions-Verhältnis beim Zweitspracherwerb Rost-Roth 2008).

Schon allein die Betrachtung dieser Untersuchungsbereiche zeigt, dass Formen und Funktionen sehr komplexe Beschreibungszusammenhänge darstellen. Zudem ergeben sich aus diesen Beschreibungen zahlreiche Hinweise auf einzelne Form-Funktions-Zusammenhänge und unterschiedliche Fragefunktionen in unterschiedlichen Kontexten.

\section{ad (2) Beschreibungen von Fragen in Grammatiken fallen in Bezug auf Form- Funktions-Bezüge oft unstimmig aus}

Grammatische Beschreibungen von Fragestrukturen gehen im allgemeinen davon aus, dass im Deutschen Entscheidungsfragen mit Verb-Erst-Stellung und Ergänzungsfragen mit Fragewort im Vorfeld als Normalfall oder Standardfall anzusehen sind. Andere Wortstellungen (VerbZweit-Stellung oder satzinterne Positionierung des Fragewortes) werden hingegen meist mit bestimmten Fragefunktionen in Zusammenhang gebracht, wie z. B. Vergewisserungsfragen, Nachfragen und Echofragen. Dasselbe gilt auch für weitere Erscheinungsformen wie selbstständige Nebensätze mit Verb-Letzt-Stellung in Fragefunktion (vgl. hierzu Rost-Roth 2006a: 133).

Unterschiede in Bezug auf die Beschreibung von Fragen zeigen sich auch in Bezug auf Bezeichnungen und Frageklassifizierungen. Hierzu einige Beispiele zu Fragedifferenzierungen und Bezeichnungen in Grammatiken:2

Engel (2004: 45) führt aus:

Es lassen sich sechs Typen von Fragen unterscheiden:

- Entscheidungsfrage

- Sachfrage

- Alternativfrage

- Rückfrage

- Gegenfrage

- Kontaktsignal (Sprecher)

[...] Die Rückfrage wird auch als "Echofrage" bezeichnet (ebd.: 47)

Helbig/Buscha (2001: 615-617) unterscheiden:

- Entscheidungsfrage ("besondere Arten":) Vergewisserungsfragen, Alternativfragen

- Ergänzungsfrage

Zifonun/Hofmann/Strecker (1997: 103-119) gehen im Kapitel zu "Quaestiven" von folgender Gliederung aus:

- Ergänzungsfrage

- Propositionale Frage (Entscheidungsfrage, Alternativfrage, Bestätigungsfrage)

- Deliberative Frage

- Nachfrage und Rückfrage

- Examensfrage und Regiefrage

Im Kapitel zu "Frage-Modi" wird zunächst von zwei Satztypen ausgegangen:

- Entscheidungsfragesatztyp

- Ergänzungsfragesatztyp (vgl. ebd.: 640)

2 Vgl. Rost-Roth (2006a: 44-49) auch zu weiteren Kategorisierungen und Klassifikationen. 
Unter Bezugnahme auf diese werden im Folgenden angeführt:

- "Aufbauende Formtypen", (Bestätigungsfragesätze, Nachfragesätze (Echofragesätze), Rückfragesätze)

sowie im Weiteren:

- Verbletzt-Formtypen

- Rhetorische Fragen (vgl. ebd.: 643-649)

In der Duden-Grammatik (2009: 889f.) werden aufgeführt:

- $w$-Fragesatz, $w$-Verbzweitsatz, $w$-Verbzweitsatz mit zusätzlichen $w$-Wörtern im Mittelfeld

- Entscheidungsfragesatz (ja/nein-Fragesätze)

- Alternativfragen

Ferner werden erwähnt: rhetorische Frage, Vergewisserungsfrage, Bestätigungsfrage, Verbletztsatz, Echofragesatz, Prüfungsfrage, deliberative Frage (ebd.)

In unterschiedlicher Weise gehen in die Bezeichnungen Fragefunktionen (Vergewisserung, Prüfung/Examen etc.) ein. Häufig finden sich auch Funktionszuschreibungen in Grammatiken. Im Folgenden ein Beispiel aus dem Duden (2009: 889f.):

Nicht alle Verbzweitsätze mit $w$-Wort im Vorfeld haben die Funktion einer Frage:

$[\ldots]$

Eine entsprechende Relativierung gilt auch für Verberstsätze:

(c) Kannst du mir mal das Salz reichen? (Aufforderungssatz)

(d) Habe ich nicht schon oft genug auf die Unmöglichkeit der Situation hingewiesen? (Ausrufesatz)

Ausrufesätze wie [...] (d) werden traditionell als "rhetorische Fragen" bezeichnet - der Sprecher erwartet hier keine wirkliche Antwort.

Umgekehrt decken die zwei Satzformen, $w$-Verbzweisatz und Verberstsatz, nicht alles ab, was der Äußerungsart "Frage" zugeordnet werden kann [...]:

Ich darf doch Schwäbisch mit Ihnen reden, nicht wahr? (Satzfunktion: Vergewisserungsfrage) Sie reichen zum ersten Mal ein solches Gesuch ein? Ihr Name ist Meier? (Satzfunktion: Bestätigungsfrage)

Auch Echofragesätze, die man äußert, wenn man etwas nicht richtig verstanden zu haben glaubt, haben diese Struktur:

Du bleibst wirklich acht Wochen in New York?

[...]

Mit Verbletztsätzen kann man nachdenkliche Fragen stellen (sogenannte deliberative Fragen) $[\ldots]$ :

Ob Anna noch kommt? Wen Otto wohl dieses Jahr einlädt? [Hervorh. im Orig. getilgt und durch meine ersetzt, M. R.-R.]

Gut erkennbar ist hier das Bestreben, das Form-Funktionsverhältnis zu verdeutlichen. Mit den Qualifizierungen als "Aufforderungssatz" bzw. "Ausrufesatz" werden Funktionen benannt; eine klare Abgrenzung zu Fragesatz als syntaktisch markierter Äußerungsform bzw. hier über Fragezeichen markierte Satzform ist jedoch über die Bezeichnung als "Satz" nicht gegeben. An anderen Stellen erscheinen Funktionsbestimmungen eingeschränkt: Echofragen erscheinen eingeschränkt auf die Funktion "nicht richtig verstehen" (auch ist der Status des Beispiels als Echofrage nicht unproblematisch, vgl. hierzu die Insertion von "wirklich"). Verb-LetztFragen erscheinen eingeschränkt auf deliberative Fragen, obgleich sie, wie im ersten Beispiel, 
Martina Rost-Roth: Formen und Funktionen von Interrogationen

auch zur Verständnissicherung eingesetzt werden können (s. u.). Unscharf erscheint zudem hier wie auch in anderen Grammatiken die Unterscheidung zwischen "Vergewisserungsfrage" und "Bestätigungsfrage" (für einen Vergleich mit der Beschreibung in der Duden-Auflage von 1998 sowie andere grammatische Beschreibungen vgl. Rost-Roth 2003 sowie Rost-Roth 2006a: 49-73, 121 u. 125).

Für die Beschreibung von Fragen allgemein zeigen sich Widersprüche in den Darstellungen zu:

- Entscheidungsfragen mit Verb-Zweit (Entsch-V2),

- Ergänzungsfragen mit Fragewort in situ (Erg-FW-SI),

- Verb-Letzt-Konstruktionen (Entsch-V-Letzt, Erg-V-Letzt).

Allein in Hinblick auf die Beschreibungen von Nachfragen zeigen sich widersprüchliche Darstellungen u. a. in Bezug auf die folgenden Punkte:

- die Verbindung zwischen bestimmten Nachfragefunktionen und sprachlichen Ausdrucksformen;

- die Syntax und Obligatorik bestimmter Strukturen (Verb-Zweit-Stellung bei Entscheidungsfragen und satzinterne Positionen von Fragewörtern in Ergänzungsfragen);

- die Auflistung und Abgrenzung verschiedener Nachfragefunktionen (Vergewisserung, Verständigungsprobleme, Erwartungsprobleme, Zeitgewinn etc.);

- die Qualifizierung von Echokomponenten und anderen Mitteln der Bezugnahme.

Ein Grund dafür, dass sich Widersprüche und Unstimmigkeiten zeigen, ist darin zu sehen, dass nicht immer deutlich zwischen Form und Funktion unterschieden wird und zudem vielfach mit Intuitionen gearbeitet wird. Viele Nachfragen sind ohne Kontext jedoch mit unterschiedlichen Intonationen und dadurch auch unterschiedlichen Funktionen vorstellbar. Zudem sind Kontexte - wie auch die folgenden Beispiele zu empirisch belegten Fragerealisierungen zeigen - schwer rekonstruierbar.

ad (3) Empirische Analysen zu gesprochener Sprache zeigen, dass vorliegende Beschreibungen Form-Funktions-Bezüge oft nicht ausreichend erfassen und das Spektrum an Fragerealisierungen nicht voll abbilden

Grundlage für die hier dargestellten empirischen Analysen sind Korpora zu unterschiedlichen Gesprächskontexten. Die Daten stammen aus unterschiedlichen Projekten und implizieren auch unterschiedliche Formalitätsgrade:

- KIH: Projekt "Kommunikationsprobleme ausländischer Studierender", Förderung durch die FNK der FU Berlin, Sonderprogramm Hochschulforschung (Kommunikation in der Hochschule Sprechstunden, Studienberatungen, Außenamt) (vgl. Rost-Roth 1998),

- VHS: Projekt "Deutschkurse für Mütter/Eltern nicht-deutscher Herkunftssprache an Berliner Schulen. Eine Studie zur Entwicklung von Ausdrucksfähigkeiten und interkulturellen Kompetenzen". Berliner Programm zur Förderung der Chancengleichheit für Frauen in Forschung und Lehre, in Zusammenarbeit mit Berliner Volkshochschulen (Mütter-/Elternkurse, Interviews, Rollenspiele) (vgl. Rost-Roth 2005),

- INF: Informelle Gespräche (Telefongespräche und gesellige Zusammenkünfte im privaten Bereich) (vgl. Rost-Roth 2006a),

- P-MoLL: "P-MoLL: Modalität in Lernervarietäten im Längsschnitt", Longitudinalstudie, experimentelles Erhebungsdesign mit verschiedenen Diskurstypen (Narrationen, Instruktionen, freie Konversationen, Argumentationen etc.) (vgl. Dittmar et al. 1990), 
- FöDaZ: DFG-Projekt "Förderunterricht und Deutsch-als-Zweitsprache-Erwerb. Eine longitudinale Untersuchung zur mündlichen Sprachkompetenz bei Schülerinnen und Schülern nicht-deutscher Herkunftssprache (ndH) in Berlin" (Ahrenholz, Lütke, RostRoth, Steinmüller) (Kinder 3. u. 4. Jahrgangsstufe) (experimentelles Erhebungsdesign, Einzelaufnahmen, Rollenspiele, Unterrichtsaufnahmen) (vgl. http://www.foedaz.unijena.de/, Ahrenholz 2006 und Rost-Roth 2006b).

In diesen Daten sind insgesamt 2691 Fragevorkommen belegt. In Hinblick auf Frageformen und syntaktische Aspekte zeigt sich, dass das strukturelle Spektrum weiter ist, als vielfach beschrieben. Die folgende Aufstellung verzeichnet die in den Daten identifizierten Strukturen und ihre Häufigkeiten. Dabei werden Nachfragen und andere Fragen getrennt betrachtet.

\begin{tabular}{|l|l|c|c|}
\hline \multicolumn{2}{|l|}{ Entscheidungsfragen (n= 477) } & $\begin{array}{l}\text { Fragen } \\
\text { (hematisch } \\
\text { weiterführend) }\end{array}$ & $\begin{array}{l}\text { Nachfragen } \\
\text { (äußerungs- } \\
\text { bezogen) }\end{array}$ \\
\hline $\begin{array}{l}\text { Verb-Erst } \\
\text { (Entsch-V1) }\end{array}$ & ?darf man eh sie 'aufnehmn? & $52,54 \%$ & $5,85 \%$ \\
\hline $\begin{array}{l}\text { Verb-Zweit } \\
\text { (Entsch-V2) }\end{array}$ & $\begin{array}{l}\text { ? und die kameras laufen jetzt } \\
\text { schon? }\end{array}$ & $18,43 \%$ & $16,66 \%$ \\
\hline $\begin{array}{l}\text { ohne Verb/elliptisch } \\
\text { (Entsch-oV) }\end{array}$ & $\begin{array}{l}\text { ?also \&lieber\& diese } \\
\text { schrift? }\end{array}$ & $7,05 \%$ & $58,55 \%$ \\
\hline $\begin{array}{l}\text { ohne Subjekt/elliptisch } \\
\text { (Entsch-oS) }\end{array}$ & ?ging? & $1,96 \%$ & $4,05 \%$ \\
\hline $\begin{array}{l}\text { Verb-Letzt } \\
\text { (Entsch-VL) }\end{array}$ & $\begin{array}{l}\text { ? ob ich da überhauptn } \\
\text { schein machen kann? }\end{array}$ & $4,70 \%$ & $1,35 \%$ \\
\hline $\begin{array}{l}\text { Vor-V2 } \\
\text { Entsch-Vor-V2) }\end{array}$ & $\begin{array}{l}\text { ?dort ham sie schon ange- } \\
\text { fang zu studiern? }\end{array}$ & $11,37 \%$ & $9,00 \%$ \\
\hline sonstige & $3,92 \%$ & $4,50 \%$ \\
\hline Summe Entscheidungsfragen & $100 \%$ & $100 \%$ \\
\hline
\end{tabular}

\begin{tabular}{|l|l|c|c|}
\hline \multicolumn{2}{|l|}{ Ergänzungsfragen (n= 265) } & & \\
\hline $\begin{array}{l}\text { FW-Satzanfang } \\
\text { (Erg-FW-SA) }\end{array}$ & ?was willst du 'drapieren? & $85,61 \%$ & $26,98 \%$ \\
\hline $\begin{array}{l}\text { FW-satzintern/final } \\
\text { (Erg-FW-SI) }\end{array}$ & ?\&wenn\& du 'was ${ }^{\wedge}$ ? & $2,15 \%$ & $11,90 \%$ \\
\hline $\begin{array}{l}\text { FW-holophrastisch } \\
\text { (Erg-FW-holo) }\end{array}$ & ?wo? & $1,43 \%$ & $53,97 \%$ \\
\hline $\begin{array}{l}\text { ohne Fragewort } \\
\text { (Erg-oFW) }\end{array}$ & ?äh für die +? & $1,43 \%$ & $3,17 \%$ \\
\hline $\begin{array}{l}\text { Fragewort-V-Letzt } \\
\text { (Erg-VL) }\end{array}$ & ?was ich dazu meine^? & $8,63 \%$ & $1,59 \%$ \\
\hline sonstige & & $0,71 \%$ & $2,38 \%$ \\
\hline Summe Ergänzungsfragen & & $100 \%$ & $100 \%$ \\
\hline
\end{tabular}

Tab. 1: Systematisierung und Frequenzen von Frageformen/Strukturtypen (vgl. Rost-Roth 2006a: 283-287 und Rost-Roth 2008) ${ }^{3}$

3 Die Auswertung stützt sich hier auf KIH-, INF- und P-MoLL-Daten. Es sind für diese Aufstellung nur die Daten von Muttersprachlern berücksichtigt. 
Martina Rost-Roth: Formen und Funktionen von Interrogationen

Neben den als Standard angesehenen Realisierungen von Verb-Erst bei Entscheidungsfragen (Entsch-V1) und Satzanfangspositionen von Fragewörtern bei Ergänzungsfragen (Erg-FWSA) sind in gesprochener Sprache weitere Fragestrukturen wie Verb-Zweitstrukturen mit Inversion bei adverbialer oder topikalisierender Vorfeldbesetzung (Entsch-Vor-V2) sowie insbesondere die Häufigkeit elliptischer Strukturen (Entsch-oV, Entsch-oS) auffällig.

Zudem ist festzuhalten, dass bei Nachfragen und anderen Fragen prinzipiell die gleichen Strukturen möglich sind. Unterschiede zeigen sich jedoch in den Frequenzen. Auffallend sind vor allem höhere Anteile von elliptischen Strukturen und V2-Strukturen bei Nachfragen.

Interessant ist, dass sich Fragerealisierungen besonders bei höflichen Fragen und Bitten syntaktisch sehr komplex gestalten können: ${ }^{4}$

D-MS: ?machen sie auch $\mathrm{n}$ schein $\operatorname{dort}^{\wedge}$ ? +

S-MS: ?ja eben das ist eben auch meine frage

ob ich da überhaupt $\mathrm{n}$ schein machen kann

wenn ich gar nicht eingeschrieben bin?

D-MS: also das is: entscheidung der dozentinnen und dozenten

(ID $1147, \mathrm{KIH})$

Hier erscheinen Verb-Letzt-Fragen in syntaktisch komplexen Gefügen mit weiteren Nebensätzen/Subordinationen. Es handelt sich oft um sog. "eingeleitete" oder "indirekte" Fragen. Eingeleitete Fragen bzw. auch "indirekte Fragen" werden beschrieben als Frageeinleitung im Hauptsatz und "eigentliche" Frage als eingebettete Frage im Nebensatz. ${ }^{5}$ Sie werden hier als Mittel für die Umsetzung von Höflichkeitsstrategien eingesetzt, die Einleitung trägt zur Indirektheit bei, ebenso wie eine Rückstufung durch die Einbettung des Nebensatzes. Zudem gibt die Frageeinleitung weiteren Raum für Modalisierungen. Kennzeichnend für den Einsatz von höflichen Fragen ist weiterhin, dass verschiedene Mittel der Modalisierung eingesetzt werden können, wie Modalverben, Konjunktiv II oder im Deutschen speziell auch Modalpartikeln (z. B. ich hätte mal ne Frage; dürfte ich kurz nachfragen, ob..., vgl. ausführlicher hierzu Rost-Roth 2004a).

\begin{tabular}{|c|c|}
\hline Frageeinleitung & "eigentliche Frage" = eingebettete Frage \\
\hline HS & NS \\
& ob-Insertion/Fragewort \\
\hline das ist auch meine Frage & ob ich da überhaupt $n$ schein machn kann \\
\hline Vorlauf & Rückstufung/Indirektheit \\
\hline \multicolumn{2}{|c|}{ Abschwächung: } \\
Modalverben (wollen, können) \\
Konjunktiv II (könnte) \\
Modalpartikeln (mal, vielleicht, denn)
\end{tabular}

Tab. 2: Sprachliche Mittel: Indirekte Fragen

4 Die Transkriptionskonventionen werden im Anhang erläutert.

5 Indirekte Fragen sind auch in der sprachwissenschaftlichen Literatur vielfach thematisiert: Wunderlich (1976: 185-189) befasst sich ausführlich mit indirekten Fragesätzen und widmet vor allem $o b$-Sätzen und Matrixsätzen Aufmerksamkeit. Mit "Fragesatzeinleitungen" und "eingeleiteten Fragesätzen" befasst sich ebenso Frier (1981: 69-71). Bei Eisenberg (1995) werden "Konjunktionalsatz und indirekter Fragesatz" sowie "indirekter Fragesatz und Relativsatz" in Hinblick auf Gemeinsamkeiten und Unterschiede diskutiert. Zaefferer (1984: 29-54) behandelt unter "Interrogativsententialen" verschiedene Formen von eingebetteten Fragen. Mit eingebetteten Interrogativsätzen ebenso wie mit selbständigen Interrogativsätzen befasst sich auch Rehbock (1991: 34-43) und Rehbein (2003) analysiert Matrixsätze in Hinblick auf die Illokution, die diesen zugeschrieben werden kann. 
Die vorausgehenden Beispiele und Analysen von empirischen Fragerealisierungen zeigen jedoch, dass die Wirklichkeit mündlicher Kommunikation und gesprochener Sprache hiermit nicht hinreichend erfasst ist. Die belegten Fragerealisierungen sind oft wesentlich komplexer, wie auch schon das vorausgehende Beispiel zeigt, insofern die Fragerealisierung einen $\mathrm{Ne}-$ bensatz (wenn....) einschließt.

Das folgende Beispiel zeigt, dass auch die Frageeinleitung als Nebensatz erscheinen kann:

S-MS: ich komm zu ihnen äh weil ich äh sie fragen wollte ob sie mich eventuell akzeptieren als prüfling im staatsexamenstudiengang $(\mathrm{h})$ ich war vor 'langer zeit mal (in nem) seminar bei ihnen + aber was ich ihnen jetzt vorschlagen wollte als thema das (äh) 'war mal ein seminar in dem ich nich war $\%$ leider\% \&da\& hat ich keine zeit \%und zwar\%= (adde1, KIH)

Den eingebetteten "eigentlichen" Fragen können auch andere Haupt- und/oder Nebensätze vorausgehen, weitere können folgen oder können auch Bestandteil derselben sein.

\begin{tabular}{|c|c|c|c|c|}
\hline $\begin{array}{c}\text { weiterer Vorlauf, } \\
\text { Begründung, } \\
\text { u. ä. }\end{array}$ & $\begin{array}{c}\text { Frage-Einleitung } \\
\text { NS }\end{array}$ & $\begin{array}{c}\text { "eigentliche Frage" } \\
\text { NS }\end{array}$ & etc. & aber \\
\hline $\begin{array}{c}\text { ich komme zu } \\
\text { ihnen }\end{array}$ & $\begin{array}{c}\text { weil } \\
\text { ich sie fragen wollte }\end{array}$ & $\begin{array}{c}\text { ob } \\
\text { zwar... }\end{array}$ \\
\hline & weitere Rückstufung & $\begin{array}{c}\text { "eigentliche" Frage = einge- } \\
\text { bettete Frage }\end{array}$ & \\
\hline
\end{tabular}

Tab. 3: Komplexere Frageformulierungen mit Frageeinleitung und eingebetteten Fragen

Gerade in institutionellen Kontexten und Formalitätsgraden, die in Hinblick auf Höflichkeitsstrategien besondere Anforderungen stellen, erfüllen Konstruktionen dieser Art weitergehende Funktionen: Im "Vorlauf" können Darstellungen von Voraussetzungen und Begründungen zur Umsetzung von Höflichkeitsstrategien beitragen, gleichzeitig wird Raum für weitere Abschwächungen und Modalisierungen eröffnet. Die Verschiebung nicht nur der eigentlichen Frage, sondern auch der Frageeinleitung "nach rechts" bzw. auf einen späteren Zeitpunkt kann funktional als weiterer Beitrag zur Erhöhung des Indirektheitsgrades analysiert werden.

Umgekehrt zeigt sich, dass Frageformulierungen aber auch syntaktisch einfacher sein können als das "Standardmodell", insofern auf Frageeinleitungen nicht immer abhängige Konstruktionen folgen müssen:

\begin{tabular}{|c|c|}
\hline Frageeinleitung & unabhängige Frage \\
HS & HS \\
\hline also das is grade meine frage & wie wolln sie's darbieten? \\
\hline
\end{tabular}

Tab. 4: Frageeinleitung mit unabhängiger Frageformulierung (HS)

Auf Frageeinleitungen können auch unabhängige Fragekonstruktionen folgen, wie im Beispiel eine Verb-Erst-Frage:

S-MS3: was wir dann ebmd vorher dann schon in der stunde (jeweils) davor dann ebmd verteiln würdn dann ?ne?

D-MS: ?ja äh also das is grade meine frage 
Martina Rost-Roth: Formen und Funktionen von Interrogationen

die ich jetzt/ die ich/äh die ich eigentlich ebm hab

die wesentlichste frage

wie/ wie wolln sie-s darbieten?

sie sagen sie wolln eingehn auf

?wolln sie das alles referiern?

S-MS1: nee nee nee $=$

S-MS3: =nein nein nein das is/

wir entwickeln einzelne thesen

(ID $1111, \mathrm{KIH}$ )

Da die Daten zeigen, dass beide Aspekte - Frageeinleitung und V-Letzt - auch unabhängig voneinander vorkommen, erscheint es sinnvoll, diese in Hinblick auf zweit- und fremdsprachliche Erwerbsverläufe, Erwerbsprobleme und Sprachvermittlung auch getrennt zu betrachten (zu entsprechenden Befunden vgl. wiederum Rost-Roth 2004b).

Auch Nachfragen gestalten sich häufig komplexer. Im nächsten Beispiel folgt auf eine syntaktisch komplexe Frage eine syntaktisch komplexe Nachfrage:

S-MS: jetzt habe ich diese ein (bewerbungsformular) bekommen

wenn er sich jetzt ehm + für ne promotion bewirbt \&ja \&

D-MS:

$\& \mathrm{mh} h \mathrm{hm} \&$

S-MS: und sie erkennen ihm ma/ magister nicht an

D-MS: ja

S-MS: ?würde das dann/ heißt es

das ganze ist abgelehnt

oder heißt es

dass sie ihn sozusagen nur zurückstufen auf magister

und (h) er noch 'um dann/ damit das dann anerkannt wird

$\&(\mathrm{~h})$ dass:

D-MS: ?\&sie meinen jetzt also dass/\&

dass er irgendein fachsemester im hauptstudium

aner/ erkannt bekommt ${ }^{\wedge}$

aber nicht das gesamte magister $+/$ ?

S-MS: jaja \&genau\&

(ID $874, \mathrm{KIH}$ )

Im Folgenden ein weiteres Beispiel, das mit einer Links-Herausstellung weitere Merkmale gesprochener Sprache mündlicher Kommunikation bei der Produktion von Fragen deutlich werden lässt:

D-MS: ?'warum jetzt in der richtung^? sie sagten journalistin \&wollen sie\&

S-MS: $\quad \& \mathrm{mh} \mathrm{hm} \mathrm{\&}$

D-MS: werden

S-MS: + ?wie meinen sie?

?die richtung journalismus

wie ich dadrauf komme?=

D-MS: ='ja

(tuc1de, KIH)

Es handelt sich hier - wie auch im vorausgehenden Beispiel - um Nachfragen, die Reformulierungen und weitergehende Explikationen von Implikationen aufweisen und Inferenzen oder Interpretationen überprüfen. Reformulierungen erscheinen hiermit neben Wiederholungen als Echokomponenten und Fragewörtern als weiteres Mittel, mit dem Bezugnahmen auf vorausgehende Äußerungen hergestellt werden können. Empirische Analysen von Nachfragen sind hierüber auch in Hinblick auf Häufigkeiten von Bezugstypen aufschlussreich. Anhand der Daten zeigen sich folgende Bezugstypen und Verteilungen: 


\begin{tabular}{|l|c|}
\hline & in Prozent $(\mathrm{n}=358)$ \\
\hline Wiederholungen (ohne Fragewörter) & $21,50 \%$ \\
\hline Wiederholungen mit Fragewörtern & $6,98 \%$ \\
\hline Fragewörter (ohne Wiederholung) & $21,78 \%$ \\
\hline Reformulierungen/Überprüfungen von Inferenzen & $34,35 \%$ \\
\hline explizite Verweise & $11,17 \%$ \\
\hline sonstige & $6,70 \%$ \\
\hline Summe (Nachfragen) (P-MoLL) & $100,00 \%{ }^{6}$ \\
\hline
\end{tabular}

Tab. 5: Häufigkeiten von Bezugstypen bei Nachfragen

Auffallend ist, dass Nachfragen, die auf Reformulierungen beruhen, einen vergleichsweise hohen Anteil haben. ${ }^{7}$ Hier zeichnen sich Zusammenhänge mit Wissenstransfer und formelleren Gesprächskontexten ab.

\section{ad (4) Formen und Funktionen von Fragen lassen sich systematischer beschreiben}

Es wird von drei Fragearten als Ausdrucksformen für unterschiedliche fragesemantische Möglichkeiten ausgegangen: Entscheidungsfragen, Ergänzungsfragen, Alternativfragen. ${ }^{8}$

Diese zeigen in Hinblick auf Syntax und Lexik jeweils unterschiedliche Erscheinungsformen, die als "Strukturtypen" in Bezug auf Verbstellung bzw. Fragewort-Positionen analysiert werden. Dabei wird analytisch eine klare Trennung der Analyse von Formen als Strukturtypen einerseits und Zuschreibungen von Äußerungsfunktionen andererseits vorgenommen.

Als weitere Unterscheidung werden vor dem Hintergrund, dass Echofragen, Nachfragen u. ä. - unabhängig von unterschiedlicher Terminologie - Beschreibungsprobleme aufwerfen, als Fragetypen nach kontextuellen Aspekten und Referenzbereichen differenziert: Fragen, die sich auf Äußerungen der Gesprächspartner als vorausgehende sprachliche Kontexte beziehen, werden als "Nachfragen" bezeichnet und als eine besondere Erscheinungsform von Fragen betrachtet. Die in der Literatur viel diskutierten Echofragen werden wiederum als Teilmenge von Nachfragen gesehen. Dementsprechend werden in Rost-Roth (2006) unterschieden:

(Fragen ( Nachfragen ( Echofragen ) ) )

Mit anderen Worten: Fragen erscheinen als übergeordneter Begriff. Nachfragen werden definiert als Fragen mit Bezugnahmen auf vorausgehende Äußerungen. Für diese lassen sich verschiedene Möglichkeiten beobachten, mit denen eine Referenz auf vorausgehende Äußerungen der Gesprächspartner hergestellt werden kann ("Bezugstypen": Fragewörter, Wiederholungen, Reformulierungen/Inferenzen und metakommunikativ explizite Verweise). Echofragen, definiert als Nachfragen, die auf Wiederholungen beruhen, werden als Teilmenge von Nachfragen gesondert aufgeführt, da sie als Nachfragen, die auf Wiederholungen basieren, syntaktisch besonderen Bedingungen unterliegen.

6 Da bei Nachfragen mehrere Möglichkeiten gleichzeitig zur Anwendung kommen können, addieren sich die Mittel bzw. entsprechenden Anteile zu mehr als $100 \%$.

Es zeigen sich auch gewisse Unterschiede in Bezug auf die Verteilung dieser Mittel in den einzelnen TeilKorpora: Nachfragen mit Reformulierungen sind in den P-MoLL-Daten und KIH-Daten häufiger als in den INF-Daten. Dies deutet darauf hin, dass ein Zusammenhang mit Gesprächskontexten besteht. Damit deuten sich insbesondere Zusammenhänge mit Aspekten wie Wissenstransfer und Formalitätsgraden an.

7 Für eine ausführliche Auseinandersetzung mit Reformulierungen und Inferenzüberprüfungen in Nachfragen vgl. Rost-Roth (2006: 199).

8 Die gesonderte Betrachtung von Alternativfragen, die zuweilen auch als besondere Erscheinung der Entscheidungsfragen gesehen werden, erweist sich u. a. auch in Hinblick auf die Beschreibung von Nachfragen als aufschlussreich (vgl. Rost-Roth 2006a: 176f., 278f.). Die folgenden Ausführungen konzentrieren sich jedoch auf Entscheidungs- und Ergänzungsfragen. 
Martina Rost-Roth: Formen und Funktionen von Interrogationen

Thematisch weiterführende Fragen, Nachfragen und Echofragen können jeweils als unterschiedliche Fragearten realisiert werden:

\begin{tabular}{|l|l|l|l|l|}
\hline & $\begin{array}{l}\text { Peter hat ein See- } \\
\text { pferd gekocht. } \\
\text { (= vorausgehende } \\
\text { Äußerung im } \\
\text { Kontext) }\end{array}$ & $\begin{array}{l}\text { Frage } \\
\text { thematisch } \\
\text { weiterfüh- } \\
\text { rend) }\end{array}$ & $\begin{array}{l}\text { Nachfrage } \\
\text { (Bezugnahme } \\
\text { auf vorausge- } \\
\text { hende Äuße- } \\
\text { rung) }\end{array}$ & $\begin{array}{l}\text { Echofrage } \\
\text { (Bezugnahme auf vo- } \\
\text { rausgehende Äußerung } \\
\text { mittels Echokomponen- } \\
\text { ten/Wiederholungen) }\end{array}$ \\
\hline $\begin{array}{l}\text { Entscheidungs- } \\
\text { fragen (Entsch) }\end{array}$ & & $\begin{array}{l}\text { und dann } \\
\text { gegessen? }\end{array}$ & $\begin{array}{l}\text { ein echtes See- } \\
\text { pferd? }\end{array}$ & ein Seepferd gekocht? \\
\hline $\begin{array}{l}\text { Ergänzungs- } \\
\text { fragen } \\
\text { (Erg) }\end{array}$ & & $\begin{array}{l}\text { und was hat } \\
\text { er dann ge- } \\
\text { macht? }\end{array}$ & $\begin{array}{l}\text { was hat der Kerl } \\
\text { gemacht }\end{array}$ & Peter hat was gekocht? \\
\hline $\begin{array}{l}\text { Alternativfragen } \\
\text { (Alt) }\end{array}$ & & $\begin{array}{l}\text { und dann } \\
\text { allein oder } \\
\text { mit andern } \\
\text { gegessen? }\end{array}$ & $\begin{array}{l}\text { ein echtes See- } \\
\text { pferd? }\end{array}$ & ein oder kein 9 \\
\hline
\end{tabular}

Tab. 6: Fragen - Nachfragen - Echofragen und Fragearten

Auch in Hinblick auf Nachfragen lassen sich weitere Systematisierungen vornehmen, wenn man Bezugstypen unterscheidet. Die Exemplifizierung erfolgt über ein von Wunderlich (1986) zur Verdeutlichung von Echofragen inspiriertes Beispiel, das hier variiert wird.

\begin{tabular}{|l|l|}
\hline $\begin{array}{l}\text { Sprachliche Mittel der Bezugnahme in } \\
\text { Nachfragen: }\end{array}$ & (Peter hat ein Seepferd gekocht = Bezugsäußerung) \\
\hline Wiederholungen (ohne Fragewörter) & Peter hat ein Seepferd gekocht? \\
\hline Wiederholungen mit Fragewörtern & $\begin{array}{l}\text { Peter hat (ein) was gekocht? } \\
\text { Wer hat ein Seepferd gekocht? }\end{array}$ \\
\hline Fragewörter (ohne Wiederholung) & wer? was? \\
\hline Reformulierungen & Er hat das Viech gegart? \\
\hline explizite Verweise & Was hast du gesagt? \\
\hline
\end{tabular}

Tab. 7: Systematisierung Bezugstypen/Nachfragen ${ }^{10}$

Eine systematische Unterscheidung von Bezugstypen scheint nicht nur in Hinblick auf die Beschreibung von Nachfragen und Frageklassifizierungen (z. B. Unterscheidung von Echofragen und anderen Nachfragen) von Bedeutung, sondern auch in Hinblick auf die Analyse von zweit- und fremdsprachlichen Erwerbsverläufen, Erwerbsproblemen und Lehrmaterialien zur Sprachförderung, insofern sich für Muttersprachler und Nichtmuttersprachler unterschiedliche Häufigkeiten beim Einsatz dieser Mittel zeigen (vgl. hierzu die Ausführungen unter These 5).

Da auch Funktionsbestimmungen für Nachfragen in der Literatur sehr unterschiedlich ausfallen, wurde auch hier eine Systematisierung auf empirischer Basis vorgenommen:

9 Da Echofragen über wörtliche Wiederholungen gekennzeichnet sind, ist im Prinzip auch vorstellbar, dass alternative Hörmöglichkeiten - z.B. bei auditiven Verstehensproblemen - thematisiert werden. Es handelt sich jedoch um einen Grenzfall, der über ein enges Verständnis von Echofragen hinausgeht.

${ }^{10}$ Bei Wiederholungen wird zudem berücksichtigt, ob sie als alleinige Mittel oder in Kombination eingesetzt werden, was sich wiederum für die Beschreibung von Echofragen als weiterführend erweist. 


\begin{tabular}{|c|c|c|c|c|}
\hline \multirow[t]{4}{*}{ I } & \multirow[t]{4}{*}{$\begin{array}{l}\text { Verständnis- } \\
\text { probleme }\end{array}$} & & $\begin{array}{l}\text { auditive/akustische Verstehenspro- } \\
\text { bleme (Hören/Wahrnehmen) }\end{array}$ & \multirow{4}{*}{$\begin{array}{l}\text { Konventionalisierung proso- } \\
\text { discher Realisierungen zur } \\
\text { Signalisierung unterschiedli- } \\
\text { cher Arten von Verstehens- } \\
\text { problemen }\end{array}$} \\
\hline & & $\mathrm{Ib}$ & $\begin{array}{l}\text { Bedeutungsverstehensprobleme (Zei- } \\
\text { chen, Dekodieren) }\end{array}$ & \\
\hline & & & $\begin{array}{l}\text { Referenzprobleme } \\
\text { (Zuordnung außersprachlicher Refe- } \\
\text { renten) }\end{array}$ & \\
\hline & & & $\begin{array}{l}\text { Interpretationsprobleme } \\
\text { propositionale Nachfragen, Inferenz- } \\
\text { überprüfungen, } \\
\text { illokutionäre Nachfragen, } \\
\text { (Rekonstruktion der } \\
\text { Bedeutung auf der Äußerungsebene, } \\
\text { Sinnzuschreibung) }\end{array}$ & \\
\hline II & \multicolumn{3}{|c|}{$\begin{array}{l}\text { Erwartungsprobleme } \\
\text { Erstaunen/ } \\
\text { Überraschung }\end{array}$} & $\begin{array}{l}\text { Sprachliche Mittel der Ebe- } \\
\text { ne I in prosodisch markierter } \\
\text { Form (Tonhöhe, Lautstärke) }\end{array}$ \\
\hline III & \multicolumn{3}{|c|}{$\begin{array}{l}\text { Weitere ("strategische") Nachfrage-Funktionen: } \\
\text { Zeitgewinn, Belustigung, Korrektur etc. }\end{array}$} & $\begin{array}{l}\text { Sprachliche Mittel der Ebe- } \\
\text { nen I + II zu anderen Zwe- } \\
\text { cken }\end{array}$ \\
\hline
\end{tabular}

Tab. 8: Systematisierung Nachfragefunktionen ${ }^{11}$

Auf eine detaillierte Beschreibung der Funktionsbereiche, muss an dieser Stelle verzichtet werden. ${ }^{12}$ Die Übersicht kann jedoch deutlich machen, wie vielfältig und komplex Funktionen allein im Bereich der Nachfragen sind. Bemerkenswert ist auch, dass alle Fragefunktionen sowohl über Ergänzungsfragen als auch über Entscheidungsfragen realisiert werden können.

Vgl. als Beispiele für auditive Verstehensprobleme (Ia) etwa:

(Peter hat ein Seepferd gekocht.) (Bezugsäußerung)

a) Peter hat ein Seepferd gekocht? $\quad$ (Ergänzungs-Nachfragen)

b) Peter hat?

c) Peter hat was?

d) bitte?

e) ein Seepferd?

f) gekocht?

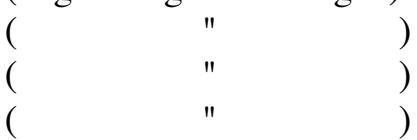

(Entscheidungs-Nachfragen)

oder als Beispiele für Bedeutungsverstehensprobleme (Ib):

(Peter hat ein Hippocampus gekocht.)

a) Was ist das?

b) ein Hippocampus?

c) Hippocampus ist Seepferd?

d) ein Seepferd?

(Bezugsäußerung)

(Ergänzungs-Nachfrage)

(Entscheidungs-Nachfrage)

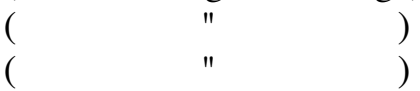

und als Beispiele für Referenzprobleme (Ic) etwa:

(Peter hat das Seepferd gekocht.)

a) Welches Seepferd?

b) das kleine Seepferd?
(Bezugsäußerung)

(Ergänzungs-Nachfrage)

(Entscheidungs-Nachfrage)

\footnotetext{
${ }^{11}$ Zur Systematisierung vgl. Rost-Roth (2006a: 257-261), zur Differenzierung der Signalisierung von Verständnisproblemen und Erwartungsproblemen in Bezug auf die auf Ebene I und II genannten prosodischen Aspekte Selting (1995).

12 Vgl. hierzu ausführlicher Rost-Roth (2006a) und Selting (1991 u. 1995).
} 
Martina Rost-Roth: Formen und Funktionen von Interrogationen

$\mathrm{Zu}$ Interpretationsproblemen (Id):

(Peter hat ein Seepferd gekocht)

a) meinst du, dass er das essen wollte?

(Bezugsäußerung)

b) wie meinst du das?

(Entscheidungs-Nachfrage)

(Ergänzungs-Nachfrage)

oder:

c) heißt das, dass er das Essen wollte (Entscheidungs-Nachfrage)

d) was soll das heißen? (Ergänzungs-Nachfrage)

und als Beispiele für Erwartungsprobleme:

(Peter hat ein Seepferd gekocht.)

a)!'Peter hat ein Seepferd gekocht?!

b)! Peter hat ein 'Seepferd gekocht?!

c)!'Wer hat ein Seepferd gekocht?!

d) ! Peter hat 'was gekocht?!

(vgl. Rost-Roth 2006a: 299-305)
(Bezugsäußerung)

(prosodisch markierte Entscheidungsfrage)

( $"$ )

(prosodisch markierte Ergänzungsfrage)

In Hinblick auf syntaktische Aspekte wurden Systematisierungen - wie auch ansonsten üblich - primär an der Verbstellung orientiert. Die Unterscheidung von Strukturtypen erfolgt auf empirischer Basis und ist bereits in Zusammenhang mit den empirischen Befunden dargestellt (vgl. hierzu die Ausführungen unter These 3). Die Systematik schließt auch verschiedene für gesprochene Sprache typische Formen wie Topikalisierungen und elliptische Frageformulierungen ein und wird im Folgenden noch einmal exemplifiziert:

\begin{tabular}{|l|l|}
\hline \multicolumn{2}{|l|}{ Entscheidungsfragen } \\
\hline Verb-Erst (Entsch-V1) & Hat Peter ein Seepferd gekocht? \\
\hline Verb-Zweit (Entsch-V2): & Peter hat ein Seepferd gekocht? \\
\hline ohne Verb/elliptisch (Entsch-oV): & ein Seepferd? \\
\hline ohne Subjekt/elliptisch (Entsch-oS) & gekocht? \\
\hline Verb-Letzt (Entsch-VL) & ob er ein Seepferd gekocht hat? \\
\hline Vor-V2 (Entsch-Vor-V2) & ein Seepferd hat er gekocht? \\
\hline Ergänzungsfragen & was hat er gekocht ? \\
\hline FW-Satzanfang (Erg-FW-SA) & er hat was gekocht?/er hat was? \\
\hline FW-satzintern/final (Erg-FW-SI) & wer? \\
\hline FW-holophrastisch (Erg-FW-holo) & er hat + ? \\
\hline ohne Fragewort (Erg-oFW) & was er gekocht hat? \\
\hline Fragewort-V-Letzt (Erg-VL)
\end{tabular}

Tab. 9: Systematisierung von Frageformen/Strukturtypen (vgl. Rost-Roth 2006: 283-287 und Rost-Roth $2008)^{13}$

\section{ad (5) Für Zweit- und Fremdspracherwerb stellen sich besondere Probleme in Hinblick auf (komplexere) Fragen}

Das folgende Beispiel von einer italienischsprachigen Muttersprachlerin (NMS-F) zeigt Probleme mit der nach $o b$ erforderlichen Nebensatz-Syntax mit Verbendstellung:

\footnotetext{
${ }^{13}$ Die Auswertung stützt sich hier auf KIH-, INF- und P-MoLL-Daten. Es sind für diese Aufstellung nur die
} Daten von Muttersprachlern berücksichtigt. 
NMS-F: ?sagst du eh:+2+ eh ob ich glaube

+ eh dass-e mein deutsch ist besser

+ eh jeden monat?

(F: 21. Mon., P-MoLL, vgl. Rost-Roth 2008: 312)

Es handelt sich hier um die komplexeste Nachfragerealisierung dieser Lernerin, belegt im 21 . Aufenthaltsmonat.

Auch in Hinblick auf die $o b$-Insertion können sich Probleme ergeben, wie hier im Beleg einer polnischen Lernerin (PM1) :

PM1: ich wollt fragn

PM1: so wenn ich + zwei \# [//] z tage länger bleibn darf-.?

EBL: \#\# zwei tage länger!

(S1 PM1 ROL tel 2-4, FöDaZ)

Vergleicht man Nachfrage Realisierungen von Nichtmuttersprachlern mit denen von Muttersprachlern, zeigen sich Beschränkungen in Bezug auf:

- die syntaktische Komplexität (Länge + Konnektoren/Subordination),

- die Lexik/Fragewörter (wofür, wonach,woran),

- die Häufigkeit von Reformulierungen,

- die Häufigkeit und Art von Frageeinleitungen (vgl. hierzu auch Rost-Roth 2004b, 2006b und 2008).

Auch in anderen Details, wie z. B. der Lexik bei reformulierenden Nachfragerealisierungen, zeigen sich Unterschiede:

\begin{tabular}{|l|c|c|}
\hline & MS (n = 51) & NMS (n = 84) \\
\hline das heißt/heißt das ${ }^{14}$ & $9,80 \%$ & $4,76 \%$ \\
\hline meinen (meinst du, du meinst u. ä.) & $11,76 \%$ & $2,38 \%$ \\
\hline also & $29,41 \%$ & $10,71 \%$ \\
\hline Summe & $100,00 \%$ & $100,00 \%$ \\
\hline
\end{tabular}

Tab. 10: Lexik in Nachfragerealisierungen

Die Unterschiede verstärken sich noch, wenn man bedenkt, dass Muttersprachler oft verschiedene dieser Mittel in komplexen Nachfrageformulierungen kombinieren. Wie bereits an anderer Stelle verdeutlicht (Rost-Roth 2008), sind aber gerade Nachfrageformulierungen für Nichtmuttersprachler von besonderer Bedeutung. Der Anteil von Nachfragen liegt in früheren Erwerbsstadien höher (70\% aller Frageformen $<\mathrm{n}=287>$ im Falle der Longitudinal-Fallstudie der o. g. italienischsprachigen Lernerin) und nimmt dann ab (unter 20\%, was den Vergleichsdaten der Muttersprachler entspricht, vgl. Rost-Roth 2008).

Einschränkungen in nichtmuttersprachlichen Kompetenzen zeigen sich auch, wenn man indirekte Fragen und Frageeinleitungen vergleicht. Hier zunächst ein Beispiel für eine muttersprachliche Frageformulierung:

D-MS: ?was kann ich für sie tun?</

S-MS: okee ich hab ne frage zur anerkennung von fernstudien (h)\

D-MS: von fernstudien $\mathrm{ja}=$

${ }^{14}$ Ein weiterer Unterschied zeigt sich bei Frageeinleitungen darin, dass diese syntaktisch assertiv oder interrogativ erscheinen können. 


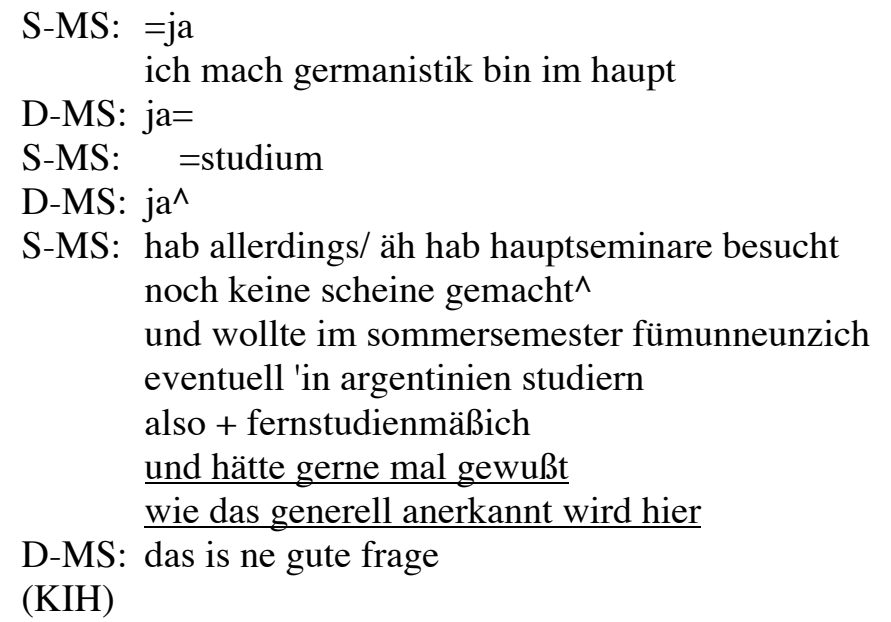

Realisiert werden hier nach einer Frageankündigung und weiteren Ausführungen eine Frageeinleitung sowie eine eingebettete Frage mit Verb-Letzt-Stellung.

Während Fragen von Muttersprachlern wie im vorausgehenden Beispiel oft sehr komplex sind, fallen Frageformulierungen von Nichtmuttersprachlern oft kürzer aus. Zudem zeigen sich nicht selten auch elliptische Formulierungen und/oder Abbrïche, wie im folgenden Beispiel:

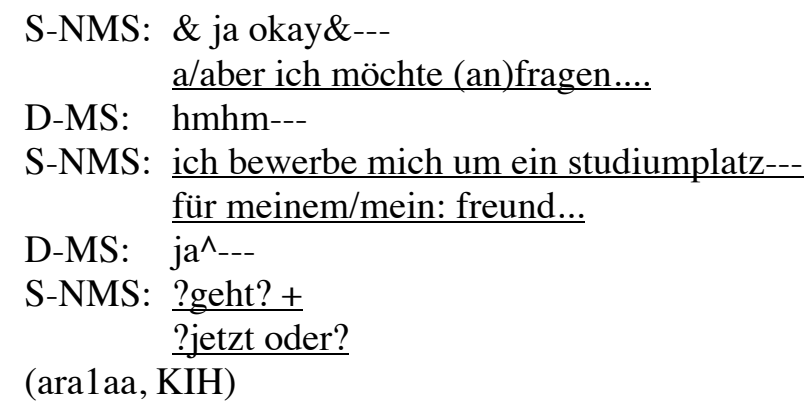

Gerade in Zusammenhang mit Höflichkeitsstrategien werden von Muttersprachlern besonders häufig Frageeinleitungen und Verb-Letzt-Konstruktionen verwendet. Die Beispiele lassen erkennen, dass Nichtmuttersprachler Schwierigkeiten haben können, entsprechende Strukturen zu produzieren, es gibt Pausen oder Abbrüche wie im vorausgehenden Beispiel oder die Strukturen "gehen nicht auf":

D-MS: und was führt sie her?

S-NMS: ah::: aso:

D-MS: ja eh suchn sie noch die ausländischen studenten

S-NMS: die auch eh die: eine (xxxxx) one-to-one eh: tutorium eh wollen?

D-MS: ja (richtig)

S-NMS: ja ich würde gerne das ja (xx) wollen (tn0korna)

S-NMS: ja ah ich hätte gerne eh mich um studienplatz eh bewerben

D-MS: $\mathrm{mh} \mathrm{hm}$

(bb2ja1, KIH)

Auch Lerner in frühen Stadien des Spracherwerbs können Probleme bei Frageformulierungen haben. Hierzu einzelne Belege für Fragerealisierungen aus dem MEK-Korpus (Integrationskurse als Mütter-/Elternkurse): 
Tür34 ?jetzt muss zu ende wa?

Ara12 ?ich frage oder lesen?

Tam10 ?die wochenende was'?

Tür34 ?wa was äh was wünschen (du denn) an den unterricht'?

Hier ist auch in einfacheren Fragen die Wortstellung in Hinblick auf Positionierungen von Verben und Fragewörtern problematisch und Frageformulierungen erfolgen oft elliptisch.

ad (6) Die Systematisierung von Frageformen und -Funktionen ist auch in Hinblick auf eine kommunikativ-pragmatisch orientierte Didaktik von Bedeutung

\section{a) Nachfragen in verständnissichernder Funktion}

Vor dem Hintergrund, dass sich für Nichtmuttersprachler ein besonderer Bedarf bei der Produktion von Fragen zeigt, ist von Interesse, wie sich die Behandlung von Nachfragen und anderen Fragen in Lehrwerken gestaltet. Im Folgenden werden einige Beispiele aus gängigen (kurstragenden) Lehrwerken gegeben.

Fragen sind bereits zentraler Bestandteil der Sprachvermittlung in den Anfangslektionen:

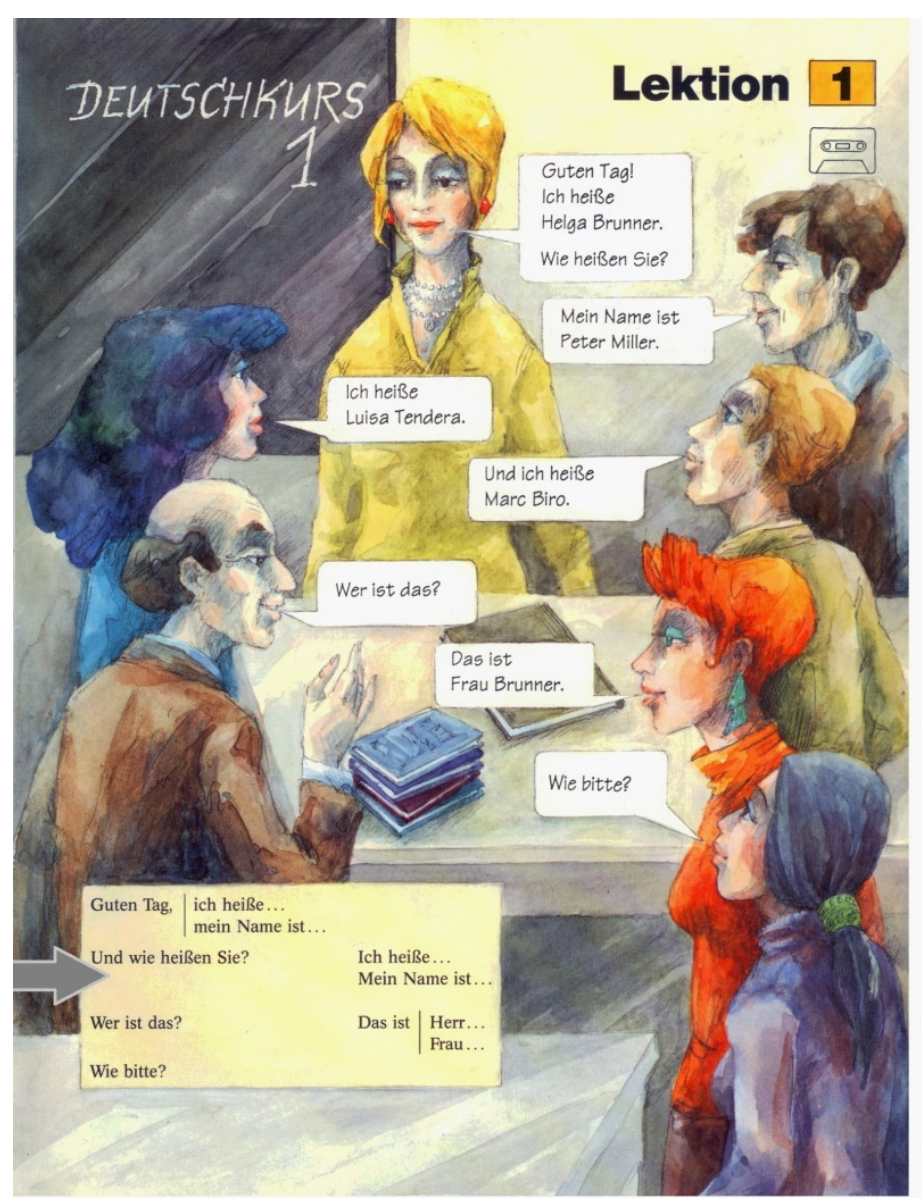

Abb. 1: Auszug aus Themen Neu (Aufderstraße 1992: 7) 
Martina Rost-Roth: Formen und Funktionen von Interrogationen

Dabei werden auch Nachfragen vermittelt; typisch ist die Vermittlung von festen Formeln wie Wie bitte?, Können Sie das bitte wiederholen? Positiv ist auch anzumerken, dass z. T. auch intonatorischen Aspekten Aufmerksamkeit gewidmet wird. ${ }^{15}$

Auch in fortgeschritteneren Lektionen und in Zusammenhang mit anderen Inhalten (wie hier zum Thema "Ämter und Behörden") lassen sich Formeln zur Verständnissicherung ausmachen. ${ }^{16}$

Wichtige Wörter und Wendungen

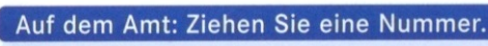

die Angehörigen • ankreuzen • anmelden • einen Antrag unterschreiben/abgeben • die Auskunft, "̈e

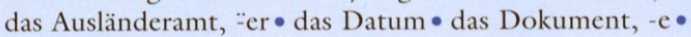
ein Formular ausfüllen/unterschreiben •

einen Moment warten • eine Nummer ziehen

\section{Das Meldeformular}

berufstätig • das Einzugsdatum • der Familienname, -n • der Familienstand • das Geburtsdatum • das Geburtsland • der Geburtsname • der Geburtsort • das Geschlecht: männlich/weiblich • die (bisherige) Hauptwohnung, - en • die Nebenwohnung, -en $\bullet$ der Ort, -e $\bullet$

die Staatsangehörigkeit, -en • die Unterschrift, -en •

der Vorname, - $\mathrm{n}$

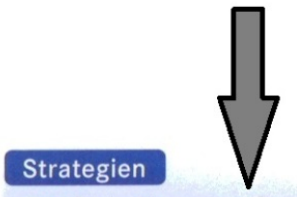

Darf ich Sie etwas fragen?

Können Sie mir helfen?

Ich brauche eine Auskunft.

Ich verstehe dieses Wort nicht.

Das habe ich nicht verstanden.

Was heißt/bedeutet das Wort?

Können Sie das bitte wiederholen?

Können Sie das bitte erklären?

Wie bitte?

Noch einmal, bitte.

Buchstabieren Sie bitte.

\section{Abb. 2: Auszug aus Schritte 2 (Bovermann/Penning-Hiemstra 2004: 23)}

Wie die Beispiele jedoch auch erkennen lassen, werden die Frageformulierungen oft nicht gesondert fokussiert (und hier deshalb als Verweis durch zusätzliche Pfeile gekennzeichnet).

Unabhängig hiervon ist festzuhalten, dass Nachfragen oft fehlen, und zwar in Kontexten, wo Sie erwartbar wären bzw. notwendig erscheinen. ${ }^{17}$ Auch Nachfragen in anderen als verständnissichernden Funktionen und vor allem auch komplexere Nachfragen sind selten zu finden. Ein Ansatz zeigt sich jedoch in Beispielen wie im Folgenden, in dem Formeln für Diskussionen vorgegeben werden:

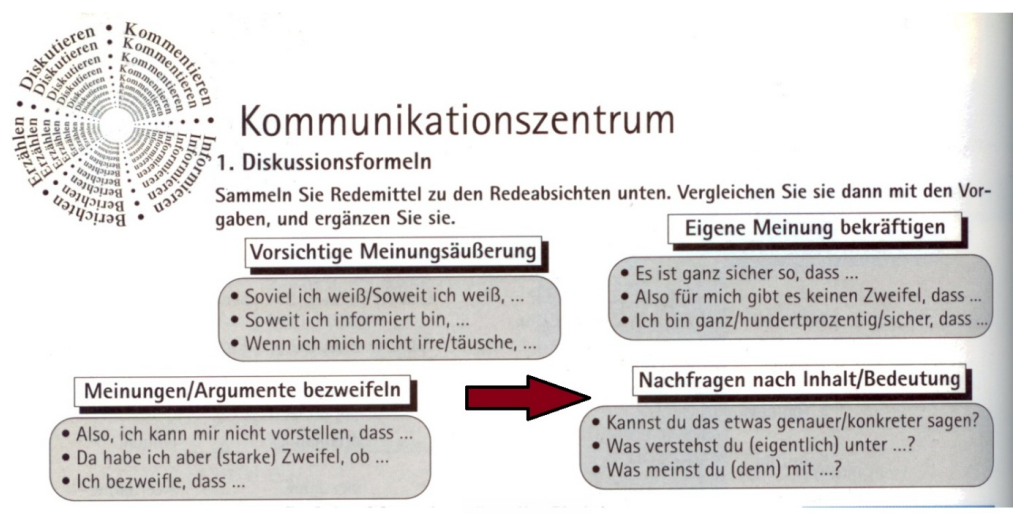

Abb. 3: Auszug aus Stufen International 3 (Vorderwülbecke 1997: 70)

\footnotetext{
15 Vgl. z. B. Moment mal! Lehrbuch 2 (Müller/Rusch/Scherling 1997) oder Stufen International 1 (Vorderwülbecke/Vorderwülbecke 2003: 13, Übung 3c; 151; ebd.: 16, Übung A 16 und A 17, Intonation Rückfrage).

${ }^{16}$ Problematisch erscheint hierbei jedoch die Bezeichnung "Strategien".

${ }^{17}$ Beispielsweise bei Wegauskünften, vgl. z. B. Eurolingua. Deutsch 1 (Funk/Koenig 1996: 108, Fragen nach dem Weg).
} 
Hier werden einige metakommunikative Formeln vermittelt. Bemerkenswert erscheint auch, dass als Bezugstypen ansonsten im allgemeinen Nachfragen mit Fragewörtern und zum Teil auch Wiederholungen erscheinen, Nachfragen mit Reformulierungen und Paraphrasierungen jedoch kaum zu beobachten sind. Wie zuvor gezeigt, ist dies jedoch gerade die Art von Nachfragen, die in den empirischen Korpusanalysen besonders häufig repräsentiert ist und zum Absichern von Verständnis und Interpretationen auch komplexerer Sachverhalte dient. Nachfragen dieser Art (vgl. Rost-Roth 2004a) sind in Lehrwerken so gut wie gar nicht zu beobachten; sie scheinen jedoch auch aus nichtmuttersprachlicher Perspektive von besonderer Bedeutung. So wird z. B. in einer Nachbesprechung im Rahmen der Datenerhebung des KIHKorpus (Kommunikation in der Hochschule) von einem japanischen Studierenden erläutert, dass er ganz bewusst versucht systematischer Verständnis abzusichern:

S-NMS: ich würde noch einmal bestätigen eh ob ich eh richtig eh verstanden hab/hatte oder nich [...] deswegen ahso eh immer wenn die deutschen mir die eh et/ eh et/eine bestimmte datum oder zei::t eh eh spricht dann wollte ich immer eeh bestätigen ob ich eh die datum oder zeit eh richtig verstanden hab verstehe oder nicht $[\ldots]$

In Hinblick auf eine Förderung von pragmatischen Kompetenzen im Bereich der Verständnissicherung könnten auch Nachfragestrategien gezielter trainiert werden. ${ }^{18}$ So ließe sich z. B. die Einführung von Nachfrageeinleitungen und -explikationen mit Hilfe von sprachlich relativ einfachen Formeln vermitteln:

\begin{tabular}{|l|l|}
\hline das heißt also, & dass $+N S$ \\
\hline & \multicolumn{1}{|c|}{$+\mathrm{HS}$} \\
\hline sie meinen also, & dass $+\mathrm{NS}$ \\
\hline & \multicolumn{1}{|c|}{$+\mathrm{HS}$} \\
\hline also & etc. \\
\hline etc. & \multicolumn{2}{|l}{} \\
\hline
\end{tabular}

Tab. 11: Komplexere Nachfragen in verständnissichernder Funktion

Einleitende Formeln wie das heißt also, Sie meinen o. ä. Art verdeutlichen nicht nur vorausgreifend die Nachfragefunktion einer folgenden Formulierung, sondern haben auch den Vorteil, einen gewissen Zeitgewinn für die anschließende Formulierung zu verschaffen. Schwierigkeiten stellen sich bei Reformulierungen von Nachfragen zudem in Hinblick auf die folgenden Aspekte: Wechsel der Deixis, syntaktische Umstellungen und auch Lexik allgemein - von daher könnten entsprechende Umformulierungsübungen auch gut mit Wortschatzübungen verbunden werden.

\section{b) Komplexe Fragen und Höflichkeitsstrategien}

Hier werden insbesondere auch indirekte Fragen, i. e. eingeleitete Frageformulierungen angeführt (Ich möchte mich erkundigen, ob..., ich möchte gerne wissen, wann...). ${ }^{19}$

Das nächste Beispiel verdeutlicht die "klassische Herangehensweise" bei der Vermittlung von indirekten Fragen:

\footnotetext{
18 Ansätze zur Förderung von (einfacheren) Nachfragestrategien finden sich beispielsweise in Sprachbrücke 1 . Lehrbuch (Mebus/Pauldrach/Rall 2000: 163, ich seh was ich seh was; ebd.: 78; ebd.: 107, Was ich gestern gemacht habe?), Stufen International 1 (Vorderwülbecke/Vorderwülbecke 2003: 104, wo vs. woher), Berliner Platz A2 (Lemcke/Rohrmann/Scherling 2004: 55, Punkt 7, Am Telefon; warum und wozu).

${ }^{19} \mathrm{Vgl}$. hierzu auch die "jugendsprachliche Adaptation" in Genial. Kursbuch A2 (Funk/Koenig/Koithan 2003: 87;

Hast Du eine Ahnung, w...).
} 
Martina Rost-Roth: Formen und Funktionen von Interrogationen

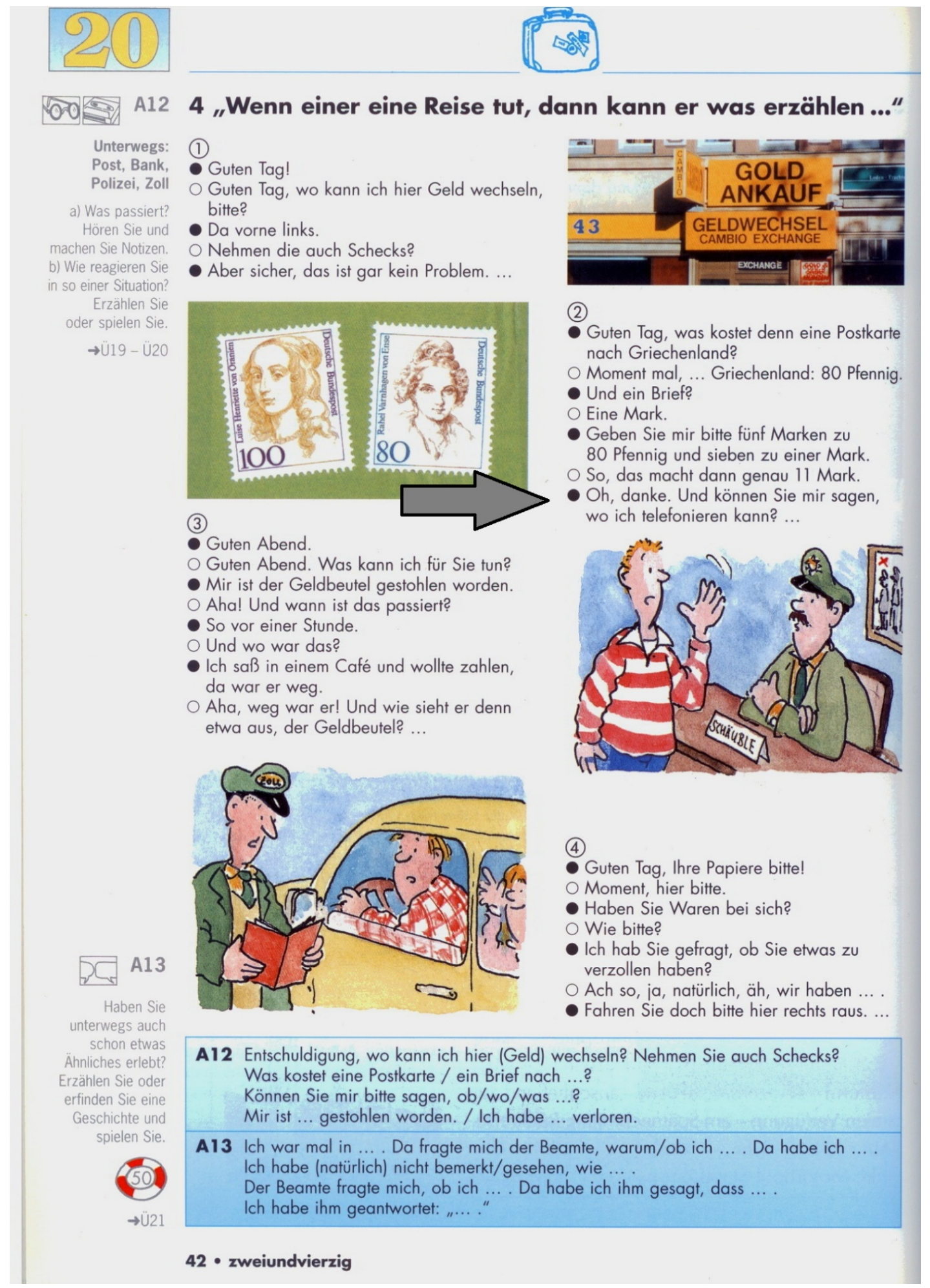

Abb.4: Auszug aus Moment mal! (Müller/Rusch/Scherling 1997: 42)

Abgesehen von den Textbeispielen mit kurzen Dialogen stehen grammatische Aspekte im Vordergrund. Die Syntax wird in Bezug auf Frageeinleitung, Konnektor (Fragewort oder $o b$ ) und Verbstellung schematisch verdeutlicht. Pragmatische Aspekte treten jedoch in den Hintergrund. Dies wird insbesondere dann deutlich, wenn man die Auflistung von Verben für die Einleitung von indirekten Fragesätzen betrachtet, mit denen sehr unterschiedliche Äußerungsfunktionen realisiert werden können: 
Indirekter Fragesatz

„Wo kann ich telefonieren?"

HAUPTSATZ $=$ WORTFRAGE (W-FRAGE)

Können Sie mir sagen, wo ich telefonieren

NEBENSATZ $=$ INDIREKTE FRAGE

"Ist das Reisen bei solchen Massen wirklich noch schön?" HAUPTSATZ = SATZFRAGE

Ich frage mich ernsthaft,

Indirekter Fragesatz möglich nach:

fragen, sich fragen, sich informieren; sagen, erzählen, beschreiben;

wissen, in den Sinn kommen, sich erinnern, verstehen;

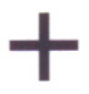

INDIREKTER FRAGESATZ bemerken, sehen, hören

\section{Fragewörter}

\section{Personen}

\begin{tabular}{|l|ll|}
\hline NOM & wer? & Wer ist das? \\
AKK & wen? & Wen siehst du? \\
DAT & wem? & Mit wem sprichst du? \\
GEN & wessen? & Wessen Buch ist das? \\
\hline
\end{tabular}

\section{Zeit}

\begin{tabular}{|ll|}
\hline wann? & Wann kommen Sie an? \\
wie lange? & Wie lange bleiben Sie? \\
wie oft? & Wie oft waren Sie schon da? \\
\hline
\end{tabular}

\section{Lokale Angaben: Position, Richtung}

\begin{tabular}{ll}
\hline wo? & Wo warst du gestern? \\
woher? & Woher kommst du gerade? \\
wohin? & Wohin fahren wir morgen? \\
\hline
\end{tabular}

\section{Sachen}

\begin{tabular}{|ll|}
\hline was? & $\begin{array}{l}\text { Was ist das? } \\
\text { Was siehst du? }\end{array}$ \\
wo(r)- + Präposition & $\begin{array}{l}\text { Womit fährst du? } \\
\text { Worauf wartest du? }\end{array}$
\end{tabular}

\section{Qualität, Quantität, Umstände}

\begin{tabular}{|ll|}
\hline wie? & Wie gut sprechen Sie Deutsch? \\
& Wie viel kostet das? \\
Wie geht es Ihnen?
\end{tabular}

Begründung: Grund, Zweck

\begin{tabular}{|ll|}
\hline $\begin{array}{l}\text { warum? } \\
\text { wozu? }\end{array}$ & Warum tust du das? \\
& Wozu brauchen Sie das? \\
\hline
\end{tabular}

fünfundvierzig • 45

Abb.5: Auszug aus Moment mal! (Müller/Rusch/Scherling 1997: 45)

Relativ selten sind Beispiele, bei denen indirekte Fragen pragmatisch in Verbindung mit Höflichkeit und als höfliche Bitten um Auskunft behandelt werden. ${ }^{20}$ Ein älteres Beispiel zeigt eine einfache, aber in Hinblick auf Form-Funktionsbezüge klare Übungsmöglichkeit:

\footnotetext{
${ }^{20}$ Vgl. hierzu beispielsweise auch Berliner Platz 3 (Köker/Lemcke 2004:11; Struktur und Übung mit Einleitungssätzen), aber auch hier bleiben die Realisierungen in Hinblick auf Häufungen von Modalisierungen und syntaktische Komplexität hinter authentischen Verwendungen und empirisch belegten Realisierungen zurück.
} 
Ü3 Formulieren Sie „höfliche“ Fragen.

Beispiel: „Wie spät ist es?" - „Könnten Sie mir sagen, / Wissen Sie, wie spät es ist?"

Aufgaben:

1. Wie komme ich zum Bahnhof? 2. Wo ist die nächste Bank? 3. Fährt dieser Bus in die City? 4. Was kostet die Fahrt zum Flughafen? 5. Wo ist die Tourist-Information? 6. Wo kann ich Geld wechseln? 7. Wie ist der
Kurs der D-Mark? 8. Wo ist das Hotel "Europa“? 9. Sind im Hotel noch Zimmer frei? 10. Was kostet ein Zimmer mit Frühstück? 11. Wo kann ich mein Gepäck lassen? 12. Wie komme ich am schnellsten in die Innenstadt?

Abb. 6: Auszug aus Deutsch aktiv (van Eunen/Geringhausen/Neuner 1989: 38)

Das folgende Beispiel zeigt, wie entsprechende Redemittel für Frage-Einleitungen als Muster zur Verfügung gestellt werden:

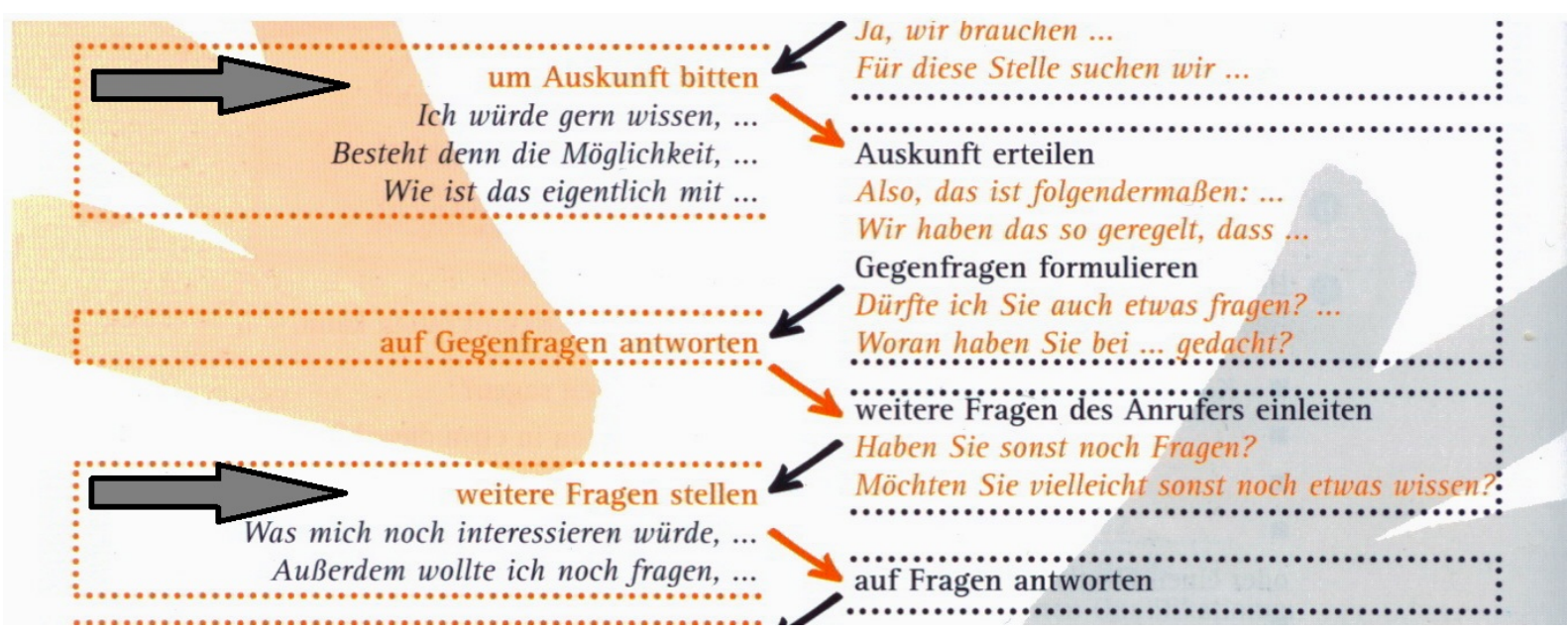

Abb. 7: Auszug aus em Neu (Perlmann-Balme/Schwalb 2005: 88)

Übungsmöglichkeiten, wie die hier dargestellten, sind jedoch eher die Ausnahme.

Mit Höflichkeit ist ein Bereich betroffen, der auch in der Literatur immer wieder als Desiderat gesehen wird. So fordern schon Erndl (1998) und Vorderwülbecke (2001) aufgrund ausführlicher Auseinandersetzung mit Lehrwerken eine stärkere Berücksichtigung von Höflichkeitsaspekten. Von besonderem Interesse ist hier die von Vorderwülbecke konzipierte dreistufige Progression für die Vermittlung von sprachlichen Realisierungen von Höflichkeit mit der "3. Stufe", in der es auch um individuelle Variation (besonders bei Bitten und Aufforderungen) und Ausdrucksformen von Höflichkeit in formelleren Situationen geht. Übungen mit dieser Zielsetzung sind immer noch eine Seltenheit. Die hier gegebenen Beispiele zeigen zudem, dass Form-Funktions-Zusammenhänge nicht immer deutlich werden.

\section{ad (7) Bestimmte Bezüge von Frageformen und Fragefunktionen sind in grammati- schen Beschreibungen und Lehrwerken (noch) unterrepräsentiert}

Abschließend kann festgehalten werden, dass zwar für einzelne Bereiche immer wieder Beispiele zu finden sind, dass aber das Spektrum bestimmte Bereiche von Nachfragefunktionen oder komplexere Frageformen nur selten oder auch gar nicht erfasst.

\section{$3 \quad$ Schlussbemerkung}

Nicht beabsichtigt ist hier eine Lehrwerkskritik. Es sollte lediglich an ausgewählten Beispielen verdeutlicht werden, wie kompliziert sich auch in Lehrwerken die Aufnahme von unterschiedlichen Frageformen und Fragefunktionen gestaltet. Vor dem Hintergrund, dass sich bereits in grammatischen und sprachwissenschaftlichen Darstellungen Beschreibungsproble- 
me zeigen, mag es nicht verwundern, dass sich auch in Lehrwerken Beschränkungen abzeichnen.

Anliegen dieses Beitrags war es, anzuregen, Beziehungen zwischen Frageformen und Fragefunktionen an der Schnittstelle von Lexik, Grammatik und Pragmatik aus sprachwissenschaftlicher Perspektive zu systematisieren und für den zweit- und fremdsprachlichen Unterricht anzuregen, die für Zweit- und Fremdsprachen-Lerner von Fremd- und Zweitsprachen besonders wichtigen Nachfragen und Fragen in Verbindung mit Höflichkeitsstrategien systematischer anzugehen.

\section{Literatur}

Altmann, Hans (1987): "Zur Problematik der Konstitution von Satzmodi als Formtypen". In: Meibauer, Jörg (Hg.): Satzmodus zwischen Grammatik und Pragmatik. Tübingen, Niemeyer: 22-56.

Ahrenholz, Bernt (2006): "Zur Entwicklung mündlicher Sprachkompetenzen bei Schülern mit Migrationshintergrund". In: ders./Apeltauer, Ernst (Hgg.): Zweitspracherwerb und curriculare Dimensionen. Empirische Untersuchungen zum Deutschlernen in Kindergarten und Grundschule. Tübingen, Stauffenburg: 91-109.

Auer, Peter (2005): "Syntax als Prozess". InLiSt - Interaction and Linguistic Structures 41. http://www.inlist.uni-bayreuth.de/issues/41/index.htm, Stand: 24.11.2011.

Austin, John L. (1962): How to do Things with Words. The William James Lectures delivered at Harvard University in 1955. Oxford: Clarendon Press.

Bäuerle, Rainer/Zimmermann, Thomas E. (1991): "Fragesätze". In: Stechow, Arnim v./Wunderlich, Dieter (Hgg.): Semantik. Ein internationales Handbuch der zeitgenössischen Forschung. Berlin/New York, de Gruyter: 333-348.

Beeching, Kate (2007): "A politeness-theoretic approach to pragmatico-semantic change". Journal of historical pragmatics 1: 69-108.

Blakemore, Diane (1994): "Echo questions: A Pragmatic Account". Lingua 94: 197-211.

Blum-Kulka, Shoshana (1987): "Indirectness and Politeness in Requests: Same or Different?" Journal of Pragmatics 11: 131-146.

Brandt, Margareta et al. (1992): "Satztyp, Satzmodus und Interrogation". In: Rosengren, Inger (Hg.): Satz und Illokution, Bd. 1. Tübingen, Niemeyer: 1-90.

Brown, Penelope/Levinson, Stephen C. (1987): Politeness. Some Universals in Language Usage. Cambridge: Cambridge University Press.

Burkhardt, Armin (1986): "Zur Phänomenologie, Typologie, Semasiologie und Onomasiologie der Frage". Deutsche Sprache 14: 23-57.

Cheon-Kostrzewa, Bok Ja/Kostrzewa, Frank (1997): "Der Erwerb der deutschen Modalpartikeln. Ergebnisse aus einer Longitudinalstudie". Deutsch als Fremdsprache 34/3: 150-155.

Clahsen, Harald/Meisel, Jürgen M./Pienemann, Manfred (1983): Deutsch als Zweitsprache. Der Spracherwerb ausländischer Arbeiter. Tübingen: Narr.

Conrad, Rudi (1978): Studien zur Syntax und Semantik von Frage und Antwort. Berlin: Akademie-Verlag.

Dittmar, Norbert u. a. (1990): "Die Erlernung modaler Konzepte des Deutschen durch erwachsene polnische Migranten. Eine empirische Längsschnittstudie". Info DaF 17/2: 125172.

Duden (1998): Die Grammatik. 6., neu bearb. Aufl., hrsg. von der Dudenredaktion. Mannheim usw.: Dudenverlag.

Duden (2009): Die Grammatik. 8., überarb. Aufl., hrsg. von der Dudenredaktion. Mannheim usw.: Dudenverlag. 
Martina Rost-Roth: Formen und Funktionen von Interrogationen

Eisenberg, Peter (1995): "Probleme der Grammatik von indirekten Fragesätzen". In: Schecker, Michael (Hg.): Fragen und Fragesätze im Deutschen. Tübingen, Stauffenburg: 141150 .

Engel, Ulrich (2004): Deutsche Grammatik. Neubearbeitung. München: Iudicium.

Erndl, Rudolf (1998): Höflichkeit im Deutschen. Konzeption zur Integration einer zentralen Gesprächskompetenz im Deutsch als Fremdsprache-Unterricht. Regensburg: FaDaF.

Felix, Sascha W. (1982): Psycholinguistische Aspekte des Zweitsprachenerwerbes. Tübingen: Narr.

Frier, Wolfgang (1981): "Zur linguistischen Beschreibung von Frage-Antwort-Zusammenhängen". In: ders. (Hg.): Pragmatik - Theorie und Praxis. Amsterdam, Rodopi: 41-91.

Gass, Susan/Neu, Joyce (Hgg.) (1996): Speech Acts Across Cultures. Challenges to Communication in a Second Language. Berlin/New York: de Gruyter.

Günthner, Susanne (2005): "Dichte Konstruktionen". InLiSt - Interaction and Linguistic Structures 43. http://www.inlist.uni-bayreuth.de/issues/43/index.htm, Stand 24.11.2011.

Hentschel, Elke (1998): Negation und Interrogation. Studien zur Universalität ihrer Funktionen. Tübingen: Niemeyer.

Helbig, Gerhard/Buscha, Joachim (2001): Deutsche Grammatik. Ein Handbuch für den Ausländerunterricht. München: Langenscheidt.

Heritage, John C./ Roth, Andrew L. (2004): "Grammar and Institution: Questions and Questioning in the Broadcast News Interview". Research on Language and Social Interaction 28/1: 1-60.

House, Juliane/Vollmer, Helmut (1988): "Sprechaktperformanz im Deutschen: zur Realisierung der Sprechhandlungen BITTEN/AUFFORDERN und SICH ENTSCHULDIGEN". Linguistische Berichte 114: 114-133.

Jefferson, Gail (1972): "Side Sequences". In: Sudnow, David (Hg.): Studies in Social Interaction. New York, Free Press: 294-338.

Kallmeyer, Werner (2005): "Struktur von Fragen und Antworten". GAIS. Gesprächsanalytisches Informationssystem: Grundbegriffe. http://gais.ids-mannheim.de/arbeitsbereich/ glossar/grundbegriffe, Stand 24.11.2011.

Kasper, Gabriele (1981): Pragmatische Aspekte in der Interimsprache. Tübingen: Narr.

Kasper, Gabriele (1990): "Linguistic Politeness. Current Research Issues". Journal of Pragmatics 14: 193-218.

Klein, Wolfgang (1982): "Einige Bemerkungen zur Frageintonation". Deutsche Sprache 10/4: 289-310.

Liedke, Martina (2007) "Was für eine Frage?! Intonatorische Strukturen als Lernproblem für Deutsch als Fremdsprache". In: Redder, Angelika (Hg): Diskurse und Texte. Festschrift für Konrad Ehlich zum 65. Geburtstag. Tübingen, Stauffenburg: 517-527

Lüger, Heinz-Helmut (1993): Routinen und Rituale in der Alltagskommunikation. Berlin/München: Langenscheidt.

Meerholz-Härle, Birgit/Tschirner, Erwin (2001): "Processability Theory: eine empirische Untersuchung". In: Aguado, Karin/Riemer, Claudia (Hgg.): Wege und Ziele. Zur Theorie, Empirie und Praxis des Deutschen als Fremdsprache (und anderer Fremdsprachen). Festschrift für Gert Henrici zum 60. Geburtstag. Baltmansweiler: Schneider Hohengehren: $155-175$.

Meibauer, Jörg (1987): "Zur Form und Funktion von Echofragen". In: Rosengren, Inger (Hg.): Sprache und Pragmatik. Lunder Symposium 1986. Stockholm, Almqvist\&Wiksell: 335-356.

Pienemann, Manfred (1998): Language Processing and Second Language Development. Processability Theory. Amsterdam/Philadelphia: John Benjamins.

Rehbein, Jochen (1999): "Zum Modus von Äußerungen". In: Redder, Angelika/ders. (Hgg.): Grammatik und mentale Prozesse. Tübingen, Stauffenburg: 91-139. 
Rehbein, Jochen (2003): "Matrix-Konstruktionen in Diskurs und Text". Zeitschrift für interkulturellen Fremdsprachenunterricht 8: 252-276.

Rehbock, Helmut (1991): "Fragen stellen - Zur Interpretation des Interrogativsatzmodus". In: Reis, Marga/Rosengren, Inger (Hgg.): Fragesätze und Fragen. Referate anläßlich der 12. Jahrestagung der Deutschen Gesellschaft für Sprachwissenschaft, Saarbrücken 1990. Tübingen, Niemeyer: 13-47.

Reis, Marga/Rosengren, Inger (Hgg.) (1991): Fragesätze und Fragen. Referate anläßlich der 12. Jahrestagung der Deutschen Gesellschaft für Sprachwissenschaft, Saarbrücken 1990. Tübingen: Niemeyer.

Roberts, Ian (Hg.) (2007): Wh-movement. London: Routledge.

Rost-Roth, Martina (unter Mitarbeit von Oliver Lechlmair) (1995): Sprachenlernen im direkten Kontakt. Autonomes Tandem in Südtirol. Eine Fallstudie. Bozen/Meran: Alpha\&Beta.

Rost-Roth, Martina (1996): "Deutsch als Fremdsprache und interkulturelle Kommunikation. Relevanzbereiche für den Fremdsprachenunterricht und Untersuchungen zu ethnographischen Besonderheiten deutschsprachiger Interaktionen im Kulturvergleich". Zeitschrift für Interkulturellen Fremdsprachenunterricht1/1.http://zif.spz.tu-darmstadt.de/jg-01-1/beitrag/ rost11.htm, Stand 24.11.2011.

Rost-Roth, Martina (1998): "Kommunikative Störungen in Beratungsgesprächen. Problempotentiale in inter- und intrakulturellen Gesprächssituationen". In: Fiehler, Reinhard (Hg.): Verständigungsprobleme und gestörte Kommunikation. Opladen, Westdeutscher Verlag: 216-244.

Rost-Roth, Martina (1999): "Der (Nicht-)Erwerb der Modalpartikeln. Eine Fallstudie zum Partikelerwerb einer italienischen Deutschlernerin mit Vergleichen zu anderen Lernervarietäten". In: Dittmar, Norbert/Giacalone Ramat, Anna (Hgg.): Grammatik und Diskurs/Grammatica e discorso. Studi sull'acquisizione dell'italiano e del tedesco/Studien zum Erwerb des Deutschen und des Italienischen. Tübingen, Stauffenburg: 165-209.

Rost-Roth, Martina (2003): "Fragen - Nachfragen - Echofragen. Formen und Funktionen von Interrogationen im gesprochenen Deutsch". Linguistik online 13/1. http://www.linguistikonline.de/13_01/rostroth.html, Stand 24.11.2011.

Rost-Roth, Martina (2004a): "Anliegensformulierungen: Aufgabenkomplexe und sprachliche Mittel. Analysen zu Anliegensformulierungen von Muttersprachlern und Nichtmuttersprachlern am Beispiel von Beratungsgesprächen und Antragsbearbeitungs-Gesprächen im Hochschulkontext". In: Baumgarten, Nicole et al. (Hgg.): Übersetzen, interkulturelle Kommunikation, Spracherwerb und Sprachvermittlung - das Leben mit mehreren Sprachen. Festschrift für Juliane House zum 60. Geburtstag. Bochum, AKS-Verlag: 186-208.

Rost-Roth, Martina (2004b): "Strukturelle Komplexität von Nachfragen: Muttersprachler und Nichtmuttersprachler im Vergleich". In: Quetz, Jürgen/Solmecke, Gert (Hgg.): Brücken schlagen: Fächer - Sprachen - Institutionen. Dokumentation zum 20. Kongress für Fremdsprachendidaktik, veranstaltet von der Deutschen Gesellschaft für Fremdsprachenforschung (DGFF), Frankfurt am Main, 1.-4. Oktober 2003. Berlin, Pädagogischer Zeitschriftenverlag: 39-49.

Rost-Roth, Martina (2005): "'Mütterkurse'. Förderung pragmatischer Kompetenzen in Deutschkursen für Frauen mit Migrationshintergrund - Lernvoraussetzungen und Kommunikationsbedürfnisse". In: Wolff, Armin/Riemer, Claudia/Neubauer, Fritz (Hgg.): Sprache lehren - Sprache lernen. Beiträge der 32. Jahrestagung des Fachverbandes Deutsch als Fremdsprache 2004. Regensburg, FaDaF: 129-154.

Rost-Roth, Martina (2006): "Intercultural Communication in Institutional Settings. Counselling Sessions". In: Bührig, Kristin/ten Thije, Jan D. (Hgg.): Beyond Misunderstanding. Linguistic analyses of intercultural communication. München, Walter Benjamins: 189216. 
Martina Rost-Roth: Formen und Funktionen von Interrogationen

Rost-Roth, Martina (2006a): Nachfragen. Formen und Funktionen äußerungsbezogener Interrogationen. Berlin/New York: de Gruyter.

Rost-Roth, Martina (2006b): "Komplexe Fragerealisierungen. SchülerInnen der 3. und 4. Grundschulklasse mit Deutsch als Zweit- und Deutsch als Muttersprache". In: Ahrenholz, Bernt (Hg.): Kinder mit Migrationshintergrund, Zweitspracherwerb und Sprachförderung. Freiburg im Breisgau, Fillibach: 241-265.

Rost-Roth, Martina (2008): "Der zweitsprachliche Erwerb der Interrogation. Theoretische Implikationen und empirische Befunde". In: Ahrenholz, Bernt u. a. (Hgg.): Empirische Forschung und Theoriebildung. Beiträge aus Soziolinguistik, Gesprochene-Sprache- und Zweitspracherwerbsforschung. Festschrift für Norbert Dittmar zum 65. Geburtstag. Frankfurt am Main, Lang: 307-321.

Searle, John R. (1974): Speech Acts. An Essay in the Philosophy of Language. Cambridge: Cambridge University Press.

Selting, Margret (1991): "w-Fragen in konversationellen Frage-Antwort-Sequenzen". In: Reis, Marga/Rosengren, Inger (Hgg.): Fragesätze und Fragen. Referate anläßlich der 12. Jahrestagung der Deutschen Gesellschaft für Sprachwissenschaft, Saarbrücken 1990. Tübingen, Niemeyer: 263-288.

Selting, Margret (1993): "Phonologie der Intonation. Probleme bisheriger Modelle und Konsequenzen einer neuen interpretativ-phonologischen Analyse". Zeitschrift für Sprachwissenschaft 1: 99-138.

Selting, Margret (1995): Prosodie im Gespräch. Aspekte einer interaktionalen Phonologie der Konversation. Tübingen: Niemeyer.

Sperber, Dan/Wilson, Deirdre (1995): Relevance. Communication and Cognition. Second Edition. Oxford: Blackwell.

Vorderwülbecke, Anne (2001): "Höflichkeit in Linguistik, Grammatik und DaF-Lehrwerk". In: Lüger, Heinz-Helmut (Hg.): Höflichkeitsstile. Frankfurt am Main usw., Lang: 28-45.

Wilson, Deirdre/Sperber, Dan (1988): "Mood and the Analysis of Non-declarative Sentences". In: Dancy, Jonathan/Moravcsik, Julius M. E./Taylor, Charles C. W. (Hgg.): $\mathrm{Hu}$ man Agency: Language, Duty and Value. Stanford, Stanford University Press: 77-101.

Wode, Henning (1976): "Some Stages in the Acquisition of Questions by Monolingual Children". Word 27, Special Issue Child Language: 261-310.

Wunderlich, Dieter (1976): Studien zur Sprechakttheorie. Frankfurt am Main: Suhrkamp.

Wunderlich, Dieter (1986): "Echofragen". Studium Linguistik 20: 44-62.

Wunderlich, Dieter (1991): Arbeitsbuch Semantik. 2., erg. Aufl. Königstein/Ts.: Athenäum.

Zaefferer, Dietmar (1984): Frageausdrücke und Fragen im Deutschen. Zu ihrer Syntax, Semantik und Pragmatik. München: Fink.

Zifonun, Gisela/ Hoffmann, Ludger/ Strecker, Bruno (1997): Grammatik der Deutschen Sprache. Berlin/New York: de Gruyter.

Zimmermann, Wolfgang (1988): Fragehandlungen und Frageverben. Ein Beitrag zur Vermittlung von Pragmatik, Grammatiktheorie und Lexikographie. Erlangen: Palm und Enke.

\section{Lehrwerke}

Aufderstraße, Hartmut (1992): Themen Neu. Lehrwerk für Deutsch als Fremdsprache. Kursbuch 1. Ismaning: Hueber.

Bovermann, Monika/Penning-Hiemstra, Sylvette (2004): Schritte 2. Deutsch als Fremdsprache. Niveau Al/2. Ismaning: Hueber.

Funk, Hermann/Koenig, Michael (1996): Eurolingua. Deutsch 1. Berlin: Cornelsen.

Funk, Hermann/Koenig, Michael/Koithan, Ute (2003): Genial. Kursbuch A2. Deutsch als Fremdsprache für Jugendliche. Berlin: Langenscheidt.

Köker, Anna/Lemcke, Christiane (2004): Berliner Platz 3. Deutsch im Alltag für Erwachsene. Zertifikatsband. Berlin/München: Langenscheidt. 
Lemcke, Christiane/Rohrmann, Lutz/Scherling, Theo (2004): Berliner Platz A2. Deutsch im Alltag für Erwachsene. Berlin: Langenscheidt.

Mebus, Gudula/Pauldrach, Andreas/Rall, Marlene (2000): Sprachbrücke 1. Lehrbuch. Stuttgart: Klett.

Müller, Martin/Rusch, Paul/Scherling, Theo (1997): Moment Mal! Lehrwerk für Deutsch als Fremdsprache. Lehrbuch 2. Berlin: Langenscheidt.

Perlmann-Balme, Michaela/Schwalb, Susanne (2005): em Neu. Hauptkurs Deutsch als Fremdsprache für die Mittelstufe. Ismaning: Hueber

van Eunen, Kees/Geringhausen, Josef/Neuner, Gerd (1989): Deutsch Aktiv Neu. Ein Lehrwerk für Erwachsene. Lehrbuch 1C. Berlin/München: Langenscheidt.

Vorderwülbecke, Anne (1997): Stufen International 3. Deutsch als Fremdsprache für Jugendliche und Erwachsene. Stuttgart: Klett.

Vorderwülbecke, Anne/Vorderwülbecke, Klaus (2003): Stufen International 1. Deutsch als Fremdsprache für Jugendliche und Erwachsene. Stuttgart usw., Klett. 
Martina Rost-Roth: Formen und Funktionen von Interrogationen

\section{Anhang: Transkriptionskonventionen}

In den Beispielen wurden in Anlehnung an P-MoLL (vgl. Dittmar et al. 1990) die folgenden Transkriptionskonventionen verwendet:

\begin{tabular}{|l|l|}
\hline+ & Pause \\
\hline$+\mathrm{N}+$ & Pause mit Sekundenangabe \\
\hline$\&$ string\& & \\
\hline string\& & gleichzeitig gesprochen \\
\hline$/$ & Abbruch \\
\hline string^ & steigende Intonation \\
\hline string_ & fallende Intonation \\
\hline @string@ & lachend gesprochen \\
\hline$\%$ string\% & leise gesprochen \\
\hline$::$ & gedehnt gesprochen \\
\hline ?string? & Markierung der Fragevorkommen \\
\hline
\end{tabular}

Bei den FöDaZ-Daten erfolgten die Transkriptionen an CA-CHAT:

\begin{tabular}{|c|c|}
\hline wird benutzt um Satzteile abzugrenzen & , (Komma) \\
\hline $\begin{array}{l}\text { text } ? \text { (am Ende der Zeile) } \\
\text { text } \mathbf{- ?}(\text { am Ende der Zeile })\end{array}$ & $\begin{array}{l}\text { ? Fragezeichen (Kennzeichnung des Endes einer } \\
\text { Äußerung) } \\
\text { wenn Tonhöhe am Ende steigend ist }\end{array}$ \\
\hline$-{ }^{\prime}$ & $\begin{array}{l}\text { Stimmhöhe steigend, steigende Kontur innerhalb } \\
\text { der Äußerung }\end{array}$ \\
\hline text [!] (in der Zeile) & Intonierung innerhalb der Äußerung als Ausruf \\
\hline text.- & $\begin{array}{l}\text { Stimmhöhe fallend, fallende Intonationskontur } \\
\text { (am Ende der Äußerung) }\end{array}$ \\
\hline text - , text & (innerhalb der Äußerung) \\
\hline text !(Am Ende einer Zeile, Ausruf) & Ausrufezeichen \\
\hline \#,\#\#,\#\#\#, ab 1 Sek. gemessen (\#2\# etc.) & Pausen \\
\hline ich [/] ich & $\begin{array}{l}\text { Wiederholungen von Äußerungsteilen ohne Verän- } \\
\text { derung }\end{array}$ \\
\hline$i c h[/ /] d u$ & $\begin{array}{l}\text { Wiederholung/Wiederaufnahmen von Äußerungs- } \\
\text { teilen mit Veränderung }\end{array}$ \\
\hline ich wollte [/-] morgen gehe ich & Abbruch/Selbstkorrektur \\
\hline$+/$ & Unterbrechung \\
\hline$+\ldots$ & 'trailing off' Abklingen (leiser werdend) \\
\hline to::r & Dehnung \\
\hline $\begin{array}{l}\text { UNTERSTREICHUNG; tor, GROSSBUCH- } \\
\text { STABEN }\end{array}$ & Betonung \\
\hline${ }^{\circ}$ text $^{\circ}$ & leiser gesprochen \\
\hline hhhhhhh & lachen \\
\hline hhhPeterhhhh & lachend gesprochen \\
\hline $\begin{array}{l}\text { Petra [?] } \\
<\text { Petra kommi }>\text { [?] }\end{array}$ & $\begin{array}{l}\text { nicht genau verständlich, vermuteter Text } \\
\text { bei mehreren Wörtern }\end{array}$ \\
\hline $\begin{array}{l}x x \\
\text { (je nach Zahl der vermuteten Wörter: xx xx) }\end{array}$ & ganz unverständlicher Text \\
\hline $\begin{array}{l}* P P P: \text { du lässt mich }[<] \text { nicht aussprechen }[>] \\
* T T T:[<] \text { auf keinen Fall }[>]\end{array}$ & Beginn und Ende von Überlappung \\
\hline
\end{tabular}

